MATHEMATICS OF COMPUTATION

Volume 69, Number 231, Pages 881-909

S 0025-5718(99)01149-7

Article electronically published on May 19, 1999

\title{
LOCAL AND PARALLEL FINITE ELEMENT ALGORITHMS BASED ON TWO-GRID DISCRETIZATIONS
}

\author{
JINCHAO XU AND AIHUI ZHOU
}

\begin{abstract}
A number of new local and parallel discretization and adaptive finite element algorithms are proposed and analyzed in this paper for elliptic boundary value problems. These algorithms are motivated by the observation that, for a solution to some elliptic problems, low frequency components can be approximated well by a relatively coarse grid and high frequency components can be computed on a fine grid by some local and parallel procedure. The theoretical tools for analyzing these methods are some local a priori and a posteriori estimates that are also obtained in this paper for finite element solutions on general shape-regular grids. Some numerical experiments are also presented to support the theory.
\end{abstract}

\section{INTRODUCTION}

In this paper, we will propose some new parallel techniques for finite element computation. These techniques are based on our understanding of the local and global properties of a finite element solution to some elliptic problems. Simply speaking, the global behavior of a solution is mostly governed by low frequency components while the local behavior is mostly governed by high frequency components. The main idea of our new algorithms is to use a coarse grid to approximate the low frequencies and then to use a fine grid to correct the resulted residue (which contains mostly high frequencies) by some local/parallel procedures.

Let us now give a somewhat more detailed but informal (and hopefully informative) description of the main ideas and results in this paper. We consider the following very simple model problem posed on a convex polygonal domain $\Omega \subset R^{2}$ :

$$
\left\{\begin{aligned}
-\Delta u+\mathbf{b} \cdot \nabla u & =f, \text { in } \Omega, \\
u & =0, \text { on } \partial \Omega .
\end{aligned}\right.
$$

The main philosophy behind this paper is that we should treat phenomena of different scales by different tools. In multigrid and domain decomposition methods, this kind of idea is used to devise iterative methods for solving a given discretization scheme (see e.g. Bank [10], Bramble [19], Chan and Mathew [22], Hackbusch [31, $\mathrm{Xu}$ [49] and Yserentant [53]); while in our approach, we try to use this type of

Received by the editor July 21, 1998.

1991 Mathematics Subject Classification. Primary 65N15, 65N30, 65N55, 65F10.

Key words and phrases. Adaptive, finite elements, local a priori and a posteriori error estimates, nonsymmetric, parallel algorithm, two-grid method.

This work was partially supported by NSF DMS-9706949, NSF ACI-9800244 and NASA NAG21236 through Penn State and Center for Computational Mathematics and Applications, The Pennsylvania State University. 
idea for designing discretization schemes. The two-grid method proposed by the first author [48, 50, 51] (and later further investigated by many others such as $33,16,24,25,33,34,42]$ ) is a result of such a consideration. The two-grid method is based on the observation that, for an equation like (1.I), the symmetric positive definite leading term $-\Delta u$ dominates the equation on high frequencies, while the low frequencies, as in a multigrid method, can be well approximated by a relatively coarse grid. Therefore, by first approximating the equation on a coarse grid, say $T^{H}(\Omega)$, we can then correct the residue (in which high frequencies dominate) on a finer grid, say $T^{h}(\Omega)$, by ignoring the lower order term and solving the resulting symmetric positive definite system.

For elliptic problems, the low frequencies are more global, while the high frequencies are more local. This fact is crucial in the multigrid methodology, in which high frequency errors are damped out by local relaxation techniques while low frequencies are handled by coarse grids. If we consider this fact more carefully, we can then imagine that if we first approximate the equation (1.1) on a coarse grid $T^{H}(\Omega)$, the residue which is dominated by high frequencies can then be resolved locally. This is precisely the central idea of the new algorithms in this paper, and is based on the local behavior of finite element approximations presented in Section 3

One technical tool for motivating this idea is the local error estimate for finite element approximations. Let $u_{h}$ be a finite element approximation to (1.1) on a quasi-uniform grid $T^{h}(\Omega)$. Then the following kind of local error estimate holds (see Theorem (3.4):

$$
\left\|u-u_{h}\right\|_{1, D} \leq C\left(\inf _{v \in S_{0}^{h}(\Omega)}\|u-v\|_{1, \Omega_{0}}+\left\|u-u_{h}\right\|_{0, \Omega}\right),
$$

where $S_{0}^{h}(\Omega)$ is the finite element space associated with $T^{h}(\Omega)$ and $D \subset \subset \Omega_{0} \subset \subset \Omega$ (here $D \subset \subset \Omega_{0}$ means that $\operatorname{dist}\left(\partial D \backslash \partial \Omega, \partial \Omega_{0} \backslash \partial \Omega\right)>0$ ).

At first glance, this type of estimate does not appear to be clearly related to what we said above, but we shall soon explain the connection. The above kind of estimate is available in the literature for quasi-uniform grids (see Nitsche and Schatz 35], Schatz [38], Schatz and Wahlbin [39, 40] and Wahlbin [46, 47]), but we need them on locally refined grids with different mesh scales, which were also discussed in [54] for local quasi-uniform grids. We are indeed able to extend these estimates to very general grids, which is one technical part of this paper-see Section 3 .

Now we consider a very special grid that is obtained by refining a given coarse grid $T^{H}(\Omega)$ for the region $\Omega_{0}$, and obtain a locally refined grid $T_{H}^{h}(\Omega)$ with mesh size $h$ in $\Omega_{0}$ and size $H$ away from $\Omega_{0}$ (see Figure 1). Let us for example consider linear finite element discretizations on this grid. Then, by (1.2) and some well-known finite element error estimates, we obtain (see Corollary [3.5)

$$
\left\|u-u_{h}\right\|_{1, D}=O\left(h+H^{2}\right) .
$$

This estimate means that we can obtain an asymptotically optimal error in the $H^{1}$ norm locally by taking $H=O(\sqrt{h})$.

With this basic result, it is then not difficult to devise a parallel algorithm on a fine grid by using a collection of overlapped subdomains. See Section 4 .

In the above approach, all the "local" solvers need to be coupled with the global coarse grid $T^{H}(\Omega)$ in some way. We can actually improve the above procedure 


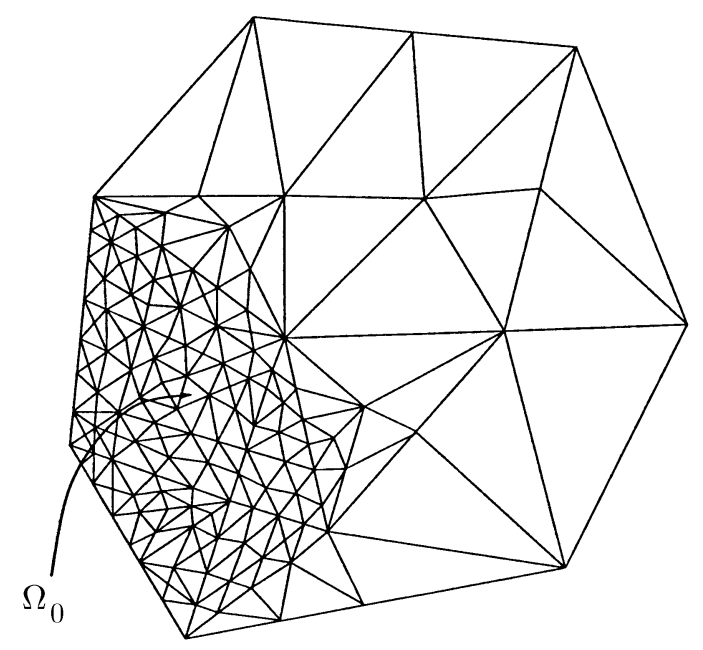

Figure 1. A local refinement mesh

by using a residue-correction technique used in $\mathrm{Xu}$ [48, 50, 51]. One prototype algorithm is as follows (see Section 4):

1. Solve on a coarse grid: Find $u_{H} \in S_{0}^{H}(\Omega)$ such that

$$
\int_{\Omega} \nabla u_{H} \cdot \nabla v+\mathbf{b} \cdot \nabla u_{H} v=\int_{\Omega} f v, \quad \forall v \in S_{0}^{H}(\Omega) .
$$

2. Correct the residue (with SPD part only) on a fine grid: Find $e_{h} \in S_{0}^{h}\left(\Omega_{0}\right)$ such that

$$
\int_{\Omega} \nabla e_{h} \cdot \nabla v=\int_{\Omega} f v-\int_{\Omega} \nabla u_{H} \nabla v-\int_{\Omega} \mathbf{b} \cdot \nabla u_{H} v, \quad \forall v \in S_{0}^{h}\left(\Omega_{0}\right) .
$$

In this algorithm, a coarse grid problem only needs to be solved once and it does not have to be coupled with the subsequence of parallel local solvers. For the above algorithm, we can still establish the following result (see Theorem 4.3):

$$
\left\|u-\left(u_{H}+e_{h}\right)\right\|_{1, D}=O\left(h+H^{2}\right) .
$$

This is a very satisfying result in many ways. As a consequence, for example, we can then design the following type of parallel algorithms: first solve the problem on a coarse grid, and then correct the residue in parallel on a collection of overlapped subdomains on a fine grid.

In practical finite element computations, it is desirable to carry out the finite element computations in an adaptive fashion, cf. Ainsworth and Oden [2], Babuška, Duran and Rodriguez [4], Babuška and Rheinboldt [5], Babuška, Zienkiewick, Gago and Oliveira [6], Bank and Weiser [15], Johnson [32] and Verfürth [45. A typical procedure is first to start with a coarse grid and then use some a posteriori estimates as a guidance to properly refine the mesh to achieve the desired accuracy. In the existing literature, a posteriori error estimates are often obtained globally, but in practical applications, they are often used locally (see e.g. [2, 4, 5, 14, 26, 27, 28, 36. 433 44, 45] and references cited therein). In this paper, we shall also present some local a posteriori error estimates that would give a certain justification of the local 
application of the a posteriori estimates. For example, we shall prove the following type of local a posteriori estimate on a general grid (see Theorem [3.9):

$$
\left\|u-u_{h}\right\|_{1, D} \leq C_{0}\left(E_{h}\left(\Omega_{0}\right)+\left\|u-u_{h}\right\|_{0, \Omega}\right)
$$

where $E_{h}\left(\Omega_{0}\right)$ is the usual a posteriori estimator on the domain $\Omega_{0}$. Again we notice that the global term $\left\|u-u_{h}\right\|_{0, \Omega}$ in the above estimate is a high order term.

More important, based on a posteriori estimates like (1.5) and a priori estimates like (1.2), we are able to design an adaptive procedure that can be carried locally in a given subdomain and hence in parallel. We believe this type of parallel adaptive techniques will have great implications in practical parallel computations.

The rest of the paper is organized as follows. In Section 2, some preliminary materials are provided. In Section 3, a number of local a priori and a posteriori error estimates are obtained for finite element discretizations on general shape regular grids. Based upon these local error estimates, several new local/parallel algorithms are devised and analyzed in Section 4 , and local and parallel adaptive processes are discussed in Section [5. In Section 6, some numerical experiments, which support our theory, are reported. Finally in Section [7 some further remarks are presented.

\section{Preliminaries}

In this section, we shall describe some basic notation and basic assumptions on the finite element spaces, and then study properties of the finite element approximation to a general linear second order elliptic boundary value problem.

Let $\Omega$ be a bounded domain in $R^{d}(d \geq 1)$. We shall use the standard notation for Sobolev spaces $W^{s, p}(\Omega)$ and their associated norms and seminorms, see e.g. [1, 23]. For $p=2$, we denote $H^{s}(\Omega)=W^{s, 2}(\Omega)$ and $H_{0}^{1}(\Omega)=\left\{v \in H^{1}(\Omega):\left.v\right|_{\partial \Omega}=0\right\}$, where $\left.v\right|_{\partial \Omega}=0$ is in the sense of trace, $\|\cdot\|_{s, \Omega}=\|\cdot\|_{s, 2, \Omega}$ and $\|\cdot\|_{\Omega}=\|\cdot\|_{0,2, \Omega}$. (In some places of this paper, $\|\cdot\|_{s, 2, \Omega}$ should be viewed as piecewise defined, if necessary.) The space $H^{-1}(\Omega)$, the dual of $H_{0}^{1}(\Omega)$, will also be used.

For $D \subset G \subset \Omega$, we write $D \subset \subset G$ to mean that $\operatorname{dist}(\partial D \backslash \partial \Omega, \partial G \backslash \partial \Omega)>0$, see Figure 2 .
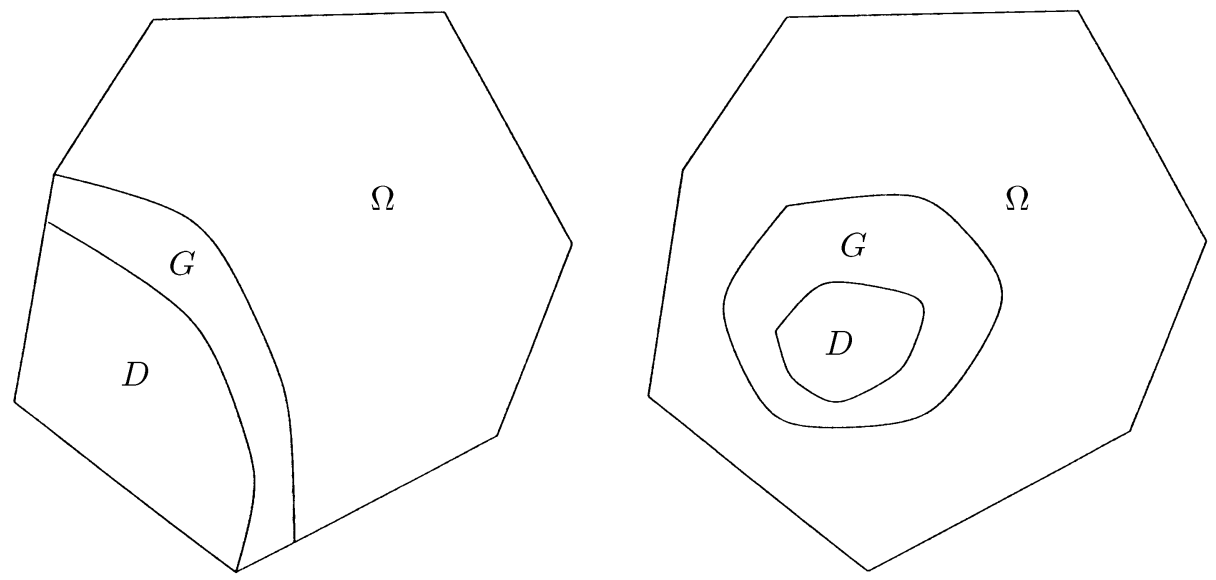

FIGURE 2. Subdomains 
Throughout this paper, we shall use the letter C (with or without subscripts) to denote a generic positive constant which may stand for different values at its different occurrences. For convenience, following [49], the symbols $\lesssim, \gtrsim$ and $\cong$ will be used in this paper. Specifically, $x_{1} \lesssim y_{1}, x_{2} \gtrsim y_{2}$ and $x_{3} \cong y_{3}$, mean that $x_{1} \leq C_{1} y_{1}, \quad x_{2} \geq c_{2} y_{2}$ and $c_{3} x_{3} \leq y_{3} \leq C_{3} x_{3}$ for some constants $C_{1}, c_{2}, c_{3}$ and $C_{3}$ that are independent of the mesh parameters.

Note that any $w \in H_{0}^{1}\left(\Omega_{0}\right)$ can be naturally extended to be a function in $H_{0}^{1}(\Omega)$ with zero outside of $\Omega_{0}$. We shall state this fact by the slightly abused notation $H_{0}^{1}\left(\Omega_{0}\right) \subset H_{0}^{1}(\Omega)$.

2.1. Finite element spaces. For generality, we will not concentrate on any specific finite element space, rather we shall study a class of finite element spaces that satisfy certain assumptions. We now describe those assumptions.

Assume that $T^{h}(\Omega)=\{\tau\}$ is a mesh of $\Omega$ with mesh-size function $h(x)$ whose value is the diameter $h_{\tau}$ of the element $\tau$ containing $x$. One basic assumption on the mesh is that it is not exceedingly over-refined locally, namely

A.0. There exists $\gamma \geq 1$ such that

$$
h_{\Omega}^{\gamma} \lesssim h(x), \quad x \in \Omega,
$$

where $h_{\Omega}=\max _{x \in \Omega} h(x)$ is the (largest) mesh size of $T^{h}(\Omega)$.

This is apparently a very mild assumption, and most practical meshes should satisfy it. Sometimes, we will drop the subscript in $h_{\Omega}$, writing $h$ for the mesh size on a domain that is clear from the context.

Associated with a mesh $T^{h}(\Omega)$, let $S^{h}(\Omega) \subset H^{1}(\Omega)$ be a finite dimensional subspace on $\Omega$ and $S_{0}^{h}(\Omega)=S^{h}(\Omega) \cap H_{0}^{1}(\Omega)$. Given $G \subset \Omega$, we define $S^{h}(G)$ and $T^{h}(G)$ to be the restriction of $S^{h}(\Omega)$ and $T^{h}(\Omega)$ to $G$, and

$$
S_{h}^{0}(G)=\left\{v \in S^{h}(\Omega): \operatorname{supp} v \subset \subset G\right\} .
$$

For any $G \subset \Omega$ mentioned in this paper, we assume that it aligns with $T^{h}(\Omega)$ when necessary.

We now state our basic assumptions on the finite element spaces.

A.1. Approximation. If $w \in H_{0}^{1}(\Omega)$, then as $h_{\Omega} \rightarrow 0$,

$$
\inf _{v \in S_{0}^{h}(\Omega)}\left(\left\|h^{-1}(w-v)\right\|_{0, \Omega}+\|w-v\|_{1, \Omega}\right)=o(1) .
$$

A.1' Approximation. There exists $r \geq 1$ such that for $w \in H_{0}^{1}(\Omega)$,

$$
\inf _{v \in S_{0}^{h}(\Omega)}\left(\left\|h^{-1}(w-v)\right\|_{0, \Omega}+\|w-v\|_{1, \Omega}\right) \lesssim\left\|h^{s} w\right\|_{1+s, \Omega}, \quad 0 \leq s \leq r .
$$

A.2. Inverse Estimate. For any $v \in S^{h}\left(\Omega_{0}\right)$,

$$
\|v\|_{1, \Omega_{0}} \lesssim\left\|h^{-1} v\right\|_{0, \Omega_{0}} .
$$

A.3. Superapproximation. For $G \subset \Omega_{0}$, let $\omega \in C_{0}^{\infty}(\Omega)$ with supp $\omega \subset \subset G$. Then for any $w \in S^{h}(G)$, there is $v \in S_{h}^{0}(G)$ such that

$$
\left\|h^{-1}(\omega w-v)\right\|_{1, G} \lesssim\|w\|_{1, G} .
$$

A.4. Trace. For any $w \in H^{1}(\tau), \tau \in T^{h}\left(\Omega_{0}\right)$,

$$
\|w\|_{0, \partial \tau} \lesssim\left\|h^{-1 / 2} w\right\|_{0, \tau}+\left\|h^{1 / 2} \nabla w\right\|_{0, \tau} .
$$

A.5. Fractional Norm. For any $G \subset \Omega$,

$$
\inf _{\chi \in S_{0}^{h}(G)}\|v-\chi\|_{1, G} \lesssim\|v\|_{1 / 2, \partial G}, \quad \forall v \in S^{h}(G) .
$$


Next we shall give an example of finite element spaces. The assumptions mentioned above are satisfied by most of the finite element spaces used in practice. We shall now briefly describe a special but popular finite element space for illustration. For simplicity, let us assume that $\Omega$ is a polygonal domain. Let $T^{h}(\Omega)$ consist of shape-regular simplices and define $S^{h}(\Omega)$ to be a space of continuous functions on $\Omega$ such that for $v \in S^{h}(\Omega), v$ restricted to each $\tau$ is a polynomial of total degree $\leq r$, namely

$$
S^{h}(\Omega)=\left\{v \in C^{1}(\bar{\Omega}):\left.v\right|_{\tau} \in P_{\tau}^{r}, \forall \tau \in T^{h}(\Omega)\right\}
$$

where $P_{\tau}^{r}$ is the space of polynomials of degree not greater than a positive integer $r$. These are the Lagrange finite element spaces, and they satisfy all the above assumptions.

The approximation assumptions A.1 and A.1' are well-known for the Lagrange finite element spaces. A.2 and A.4 are well-known and they can be easily proven by a standard scaling argument. The superapproximation assumption has been discussed in many papers, cf. [35, 39] 40, 46, 47]. This superapproximation assumption can be easily verified for the Lagrange finite element spaces (2.8), using a locally defined interpolation operator $I_{h}$ satisfying

$$
\left\|\phi-I_{h} \phi\right\|_{0, \infty, \tau} \lesssim\left|h^{r} \phi\right|_{r, \infty, \tau}
$$

where $|\cdot|_{r, \infty, \Omega}$ denotes the $r^{t h}$ semi-norm involving only $r^{t h}$ derivatives. Setting $\phi=\omega w$ in (2.9) and noting that $r^{t h}$ derivatives of $w$ vanish, and using the inverse estimates, one obtains Assumption A.3.

The verification of A.5 can go as follows. For $v \in S^{h}(G)$, let $\chi \in S_{0}^{h}(G)$ be the unique function satisfying

$$
(\nabla \chi, \nabla \phi)=(\nabla v, \nabla \phi), \quad \forall \phi \in S_{0}^{h}(G) .
$$

Then $v-\chi$ is discrete harmonic. The desired result then follows from the following well-known (cf. [52]) estimate for discrete harmonic functions:

$$
\|v-\chi\|_{1, G} \lesssim\|v-\chi\|_{1 / 2, \partial G}=\|v\|_{1 / 2, \partial G} .
$$

2.2. A model problem. In this subsection, we shall study some basic properties of general second order elliptic boundary value problems and their finite element approximations. We consider the homogeneous boundary value problem

$$
\left\{\begin{aligned}
L u & =f, & & \text { in } \Omega, \\
u & =0, & & \text { on } \partial \Omega .
\end{aligned}\right.
$$

Here $L$ is a general linear second order elliptic operator:

$$
L u=-\sum_{i, j=1}^{d} \frac{\partial}{\partial x_{j}}\left(a_{i j} \frac{\partial u}{\partial x_{i}}\right)+\sum_{i=1}^{d} b_{i} \frac{\partial u}{\partial x_{i}}+c u,
$$

satisfying $a_{i j}, b_{i} \in W^{1, \infty}(\Omega), c \in L^{\infty}(\Omega)$, and $\left(a_{i j}\right)$ is uniformly positive definite on $\Omega$.

The weak form of (2.10) is as follows: Find $u \equiv L^{-1} f \in H_{0}^{1}(\Omega)$ such that

$$
a(u, v)=(f, v), \quad \forall v \in H_{0}^{1}(\Omega),
$$

where $(\cdot, \cdot)$ is the standard inner-product of $L^{2}(\Omega)$ and

$$
a(u, v)=a_{0}(u, v)+N(u, v)
$$


with

$$
a_{0}(u, v)=\int_{\Omega} \sum_{i, j=1}^{d} a_{i j} \frac{\partial u}{\partial x_{i}} \frac{\partial v}{\partial x_{j}} \text { and } \quad N(u, v)=\int_{\Omega} \sum_{i=1}^{d} b_{i} \frac{\partial u}{\partial x_{i}} v+c u v .
$$

Note that

$$
\|w\|_{1, \Omega}^{2} \lesssim a_{0}(w, w), \quad \forall w \in H_{0}^{1}(\Omega)
$$

and

$$
a_{0}(u, v) \lesssim\|u\|_{1, \Omega}\|v\|_{1, \Omega}, \quad N(u, v) \lesssim\|u\|_{0, \Omega}\|v\|_{1, \Omega}, \quad \forall u, v \in H_{0}^{1}(\Omega) .
$$

Our basic assumption is that (2.11) is well-posed, namely 2.11) is uniquely solvable for any $f \in H^{-1}(\Omega)$. (A simple sufficient condition for this assumption to be satisfied is that $c \geq 0$.) An application of the open-mapping theorem yields

$$
\|w\|_{1, \Omega} \lesssim\|L w\|_{-1, \Omega}, \quad \forall w \in H_{0}^{1}(\Omega) .
$$

It is easy to see that if $L$ satisfies the above assumption and the above estimates, so does its formal adjoint

$$
L^{*} u=-\sum_{i, j=1}^{d} \frac{\partial}{\partial x_{i}}\left(a_{i j} \frac{\partial u}{\partial x_{j}}\right)-\sum_{i=1}^{d} \frac{\partial\left(b_{i} u\right)}{\partial x_{i}}+c u .
$$

A sufficient and necessary condition for the well-posedness of (2.11) is that

$$
\|w\|_{1, \Omega} \lesssim \sup _{\phi \in H_{0}^{1}(\Omega)} \frac{a(w, \phi)}{\|\phi\|_{1, \Omega}}, \quad \forall w \in H_{0}^{1}(\Omega)
$$

and

$$
\|w\|_{1, \Omega} \lesssim \sup _{\phi \in H_{0}^{1}(\Omega)} \frac{a(\phi, w)}{\|\phi\|_{1, \Omega}}, \quad \forall w \in H_{0}^{1}(\Omega) .
$$

We have (cf. [30]) the following estimate for the regularity of the solution of (2.10) or (2.11):

$$
\|u\|_{1+\alpha, \Omega} \lesssim\|f\|_{-1+\alpha, \Omega}
$$

for some $\alpha \in(0,1]$ depending on $\Omega$ and the coefficients of $L$.

For some $G \subset \Omega$, we need the following assumption.

$\mathbf{R}(\mathbf{G})$. For any $f \in L^{2}(G)$, there exists a $w \in H_{0}^{1}(G)$ satisfying

$$
a(v, w)=(f, v), \forall v \in H_{0}^{1}(G),
$$

and

$$
\|u\|_{1+\alpha, G} \lesssim\|f\|_{-1+\alpha, G} .
$$

It is well-known (cf. [51]) that if $h_{\Omega} \ll 1$ (depending on $N$ ) and Assumption A.1 holds, then

$$
\left\|w_{h}\right\|_{1, \Omega} \lesssim \sup _{\phi \in S_{0}^{h}(\Omega)} \frac{a\left(w_{h}, \phi\right)}{\|\phi\|_{1, \Omega}}, \quad \forall w_{h} \in S_{0}^{h}(\Omega)
$$

and

$$
\left\|w_{h}\right\|_{1, \Omega} \lesssim \sup _{\phi \in S_{0}^{h}(\Omega)} \frac{a\left(\phi, w_{h}\right)}{\|\phi\|_{1, \Omega}}, \quad \forall w_{h} \in S_{0}^{h}(\Omega)
$$

Throughout this paper, we will assume that $h_{\Omega} \ll 1$ (when $N(\cdot, \cdot) \neq 0$ ) and Assumption A.1 holds, so that the above two estimates hold. From the above two 
estimates, we can then define Galerkin-projections $P_{h}\left(\equiv P_{h}^{\Omega}\right): H_{0}^{1}(\Omega) \mapsto S_{0}^{h}(\Omega)$ and $P_{h}^{*}\left(\equiv\left(P_{h}^{\Omega}\right)^{*}\right): H_{0}^{1}(\Omega) \mapsto S_{0}^{h}(\Omega)$ by

$$
a\left(u-P_{h} u, v\right)=0 \quad \text { and } \quad a\left(v, u-P_{h}^{*} u\right)=0, \quad \forall v \in S_{0}^{h}(\Omega),
$$

and apparently

$$
\left\|P_{h} u\right\|_{1, \Omega} \lesssim\|u\|_{1, \Omega} \quad \text { and } \quad\left\|P_{h}^{*} u\right\|_{1, \Omega} \lesssim\|u\|_{1, \Omega}, \quad \forall u \in H_{0}^{1}(\Omega) .
$$

From (2.17), various a global priori error estimates can be obtained from the approximate properties of the finite element subspaces $S^{h}(\Omega)$ (cf. [23]). Particularly, by Assumption A.1, if $u \in H_{0}^{1}(\Omega)$, then

$$
\left\|u-P_{h} u\right\|_{1, \Omega}=o(1) \text { and }\left\|u-P_{h}^{*} u\right\|_{1, \Omega}=o(1), \quad \text { as } h_{\Omega} \rightarrow 0 .
$$

Now we introduce the following quantity:

$$
\rho_{\Omega}(h)=\max \left(\rho_{\Omega, L}(h), \rho_{\Omega, L^{*}}(h)\right),
$$

where

$$
\rho_{\Omega, L}(h)=\sup _{f \in L^{2}(\Omega),\|f\|_{0, \Omega}=1} \inf _{v \in S_{0}^{h}(\Omega)}\left\|L^{-1} f-v\right\|_{1, \Omega} .
$$

Similarly, if Assumption $\mathrm{R}(\mathrm{G})$ holds, we can define $\rho_{G}(h)$ well.

Lemma 2.1. Assume that $h_{\Omega} \ll 1$ and Assumption A.1 holds. Then

$$
\left\|\left(I-P_{h}\right) L^{-1} f\right\|_{1, \Omega} \lesssim \rho_{\Omega}(h)\|f\|_{0, \Omega}, \quad \forall f \in L^{2}(\Omega),
$$

and

$$
\left\|u-P_{h} u\right\|_{0, \Omega} \lesssim \rho_{\Omega}(h)\left\|u-P_{h} u\right\|_{1, \Omega} \quad \forall u \in H_{0}^{1}(\Omega) .
$$

Proof. Let $u=L^{-1} f \in H_{0}^{1}(\Omega)$. For any $\phi \in H_{0}^{1}(\Omega)$, we have

$$
a\left(u-P_{h} u, \phi\right)=a\left(u-v, \phi-P_{h}^{*} \phi\right), \quad \forall v \in S_{0}^{h}(\Omega) .
$$

Thus, (2.13) and (2.17) yield

$$
\left\|u-P_{h} u\right\|_{1, \Omega} \lesssim\|u-v\|_{1, \Omega}, \quad \forall v \in S_{0}^{h}(\Omega),
$$

which produce (2.19).

To prove (2.20), we use the Aubin-Nitsche duality argument. For each $\phi \in L^{2}(\Omega)$, let $w=\left(L^{*}\right)^{-1} \phi \in H_{0}^{1}(\Omega)$. Then

$$
\left\|w-P_{h}^{*} w\right\|_{1, \Omega} \lesssim \rho_{\Omega}(h)\|\phi\|_{0, \Omega} .
$$

Note that

$$
\begin{aligned}
\left|\left(u-P_{h} u, \phi\right)\right| & =\left|a\left(u-P_{h} u, w\right)\right|=\left|a\left(u-P_{h} u, w-P_{h}^{*} w\right)\right| \\
& \lesssim\left\|u-P_{h} u\right\|_{1, \Omega}\left\|w-P_{h}^{*} w\right\|_{1, \Omega} .
\end{aligned}
$$

We get (2.20) from combining (2.22), the above inequalities, and

$$
\left\|u-P_{h} u\right\|_{0, \Omega}=\sup _{\phi \in L^{2}(\Omega),\|\phi\|_{0, \Omega}=1}\left(u-P_{h} u, \phi\right) .
$$


Lemma 2.2. Assume that $h_{\Omega} \ll 1$ and Assumption A.1 holds. Then $\rho_{\Omega}(h)=o(1)$ as $h_{\Omega} \rightarrow 0$. Moreover,

$$
\rho_{\Omega}(h) \lesssim h_{\Omega}^{\alpha}
$$

provided

$$
\inf _{v \in S_{0}^{h}(\Omega)}\|w-v\|_{1, \Omega} \lesssim h_{\Omega}^{\alpha}\|w\|_{1+\alpha, \Omega} .
$$

Proof. It is easy to see that $L^{-1}: L^{2}(\Omega) \rightarrow H_{0}^{1}(\Omega)$ is compact. Hence, $P_{h} L^{-1}$ : $L^{2}(\Omega) \mapsto H_{0}^{1}(\Omega)$ is a compact and continuous mapping. Since $\forall f \in L^{2}(\Omega)$

$$
\left\|\left(I-P_{h}\right) L^{-1} f\right\|_{1, \Omega} \rightarrow 0, \quad \text { as } h_{\Omega} \rightarrow 0,
$$

we get

$$
\sup _{f \in L^{2}(\Omega),\|f\|_{0, \Omega}=1}\left\|\left(I-P_{h}\right) L^{-1} f\right\|_{1, \Omega} \rightarrow 0, \quad \text { as } h_{\Omega} \rightarrow 0,
$$

which implies $\rho_{\Omega}(h)=o(1)$ as $h_{\Omega} \rightarrow 0$.

(2.23) is immediately obtained from (2.21), (2.15) and (2.24).

Remark 2.3. We would like to point out that the first part of Lemma 2.2 generalizes [33].

\section{LOCAL A PRIORI AND A POSTERIORI ERROR ESTIMATES}

In this section, we shall present a number of local a priori and a posteriori error estimates for finite element discretizations on general shape regular grids. Novelties of our estimates lie in, for example, the weak assumption on the underlying grids as well as the generality of model continuous problems. Although these general estimates are theoretically interesting in their own right, our main motivation is to use them to devise and analyze some new local/parallel methods to be presented in the following sections.

3.1. Local a priori error estimates. The results presented here generalize local a priori error estimates known in the literature [35, 39, 40, 46, 47, 54, to more general second order differential equations and more general finite element meshes.

First, we need the following technical result.

Lemma 3.1. Let $D \subset \subset \Omega_{0}$, and let $\omega \in C_{0}^{\infty}(\Omega)$ be such that supp $\omega \subset \subset \Omega_{0}$. Then

$$
a_{0}(\omega w, \omega w) \leq 2 a\left(w, \omega^{2} w\right)+C\|w\|_{0, \Omega_{0}}^{2}, \quad \forall w \in H_{0}^{1}(\Omega) .
$$

Proof. From the identity

$$
\begin{aligned}
a_{0}(\omega w, \omega w)= & a_{0}\left(w, \omega^{2} w\right) \\
& +\int_{\Omega} \sum_{i, j=1}^{d} a_{i j}\left(\left(\frac{\partial \omega}{\partial x_{i}} \frac{\partial(\omega w)}{\partial x_{j}}-\frac{\partial \omega}{\partial x_{j}} \frac{\partial(\omega w)}{\partial x_{i}}\right) w+\frac{\partial \omega}{\partial x_{i}} \frac{\partial \omega}{\partial x_{j}} w^{2}\right),
\end{aligned}
$$

we get

$$
a_{0}(\omega w, \omega w) \leq a_{0}\left(w, \omega^{2} w\right)+C\left(\|\omega w\|_{1, \Omega}\|w\|_{0, \Omega_{0}}+\|w\|_{0, \Omega_{0}}^{2}\right) .
$$

Note that

$$
a_{0}\left(w, \omega^{2} w\right)=a\left(w, \omega^{2} w\right)-N(\omega w, \omega w)+\int_{\Omega} \sum_{j=1}^{d} b_{j} \frac{\partial \omega}{\partial x_{j}} \omega w^{2}
$$


and

$$
\|\omega w\|_{1, \Omega}^{2} \lesssim a_{0}(\omega w, \omega w)
$$

so we have

$$
\begin{aligned}
a_{0}(\omega w, \omega w) & \leq a\left(w, \omega^{2} w\right)+C\left(\|\omega w\|_{1, \Omega}\|w\|_{0, \Omega_{0}}+\|w\|_{0, \Omega_{0}}^{2}\right) \\
& \leq a\left(w, \omega^{2} w\right)+\frac{1}{2} a_{0}(\omega w, \omega w)+C\|w\|_{0, \Omega_{0}}^{2} .
\end{aligned}
$$

An application of a kick-back argument then leads to (3.1).

We shall now present a local a priori estimate for finite element approximation that will play a crucial role in our analysis. This type of estimates can be found in [35, 39, 40, 46, 47]; a new feature here is the generality of the underlying finite element grid for which this estimate is proven valid.

Lemma 3.2. Suppose that $f \in H^{-1}(\Omega)$ and $D \subset \subset \Omega_{0}$. If Assumptions A.0, A.2 and $A .3$ hold and $w \in S^{h}\left(\Omega_{0}\right)$ satisfies

$$
a(w, v)=f(v), \quad \forall v \in S_{h}^{0}\left(\Omega_{0}\right),
$$

then

$$
\|w\|_{1, D} \lesssim\|w\|_{0, \Omega_{0}}+\|f\|_{-1, \Omega_{0}}
$$

where

$$
\|f\|_{-1, \Omega_{0}}=\sup _{\phi \in H_{0}^{1}\left(\Omega_{0}\right),\|\phi\|_{1, \Omega_{0}}=1} f(v) .
$$

Proof. Let $p$ be an integer such that $p \geq 2 \gamma-1$, and let $\Omega_{j}(j=1,2, \cdots, p)$ satisfy

$$
D \subset \subset \Omega_{p} \subset \subset \Omega_{p-1} \subset \subset \cdots \subset \subset \Omega_{1} \subset \subset \Omega_{0} .
$$

Choose $D_{1} \subset \Omega$ satisfying $D \subset \subset D_{1} \subset \subset \Omega_{p}$ and $\omega \in C_{0}^{\infty}(\Omega)$ such that $\omega \equiv 1$ on $\bar{D}_{1}$ and $\operatorname{supp} \omega \subset \subset \Omega_{p}$. Then, from Assumption A.3, there exists $v \in S_{h}^{0}\left(\Omega_{p}\right)$ such that

$$
\left\|\omega^{2} w-v\right\|_{1, \Omega_{p}} \lesssim h_{\Omega_{0}}\|w\|_{1, \Omega_{p}}
$$

which implies

$$
a\left(w, \omega^{2} w-v\right) \lesssim h_{\Omega_{0}}\|w\|_{1, \Omega_{p}}^{2}
$$

and

$$
\begin{aligned}
|f(v)| & \lesssim\|f\|_{-1, \Omega_{0}}\|v\|_{1, \Omega_{p}} \\
& \lesssim\|f\|_{-1, \Omega_{0}}\left(h_{\Omega_{0}}\|w\|_{1, \Omega_{p}}+\|\omega w\|_{1, \Omega}\right) .
\end{aligned}
$$

Since $v \in S_{h}^{0}\left(\Omega_{p}\right) \subset S_{h}^{0}\left(\Omega_{0}\right)$, (3.2) implies

$$
a\left(w, \omega^{2} w\right)=a\left(w, \omega^{2} w-v\right)+f(v) .
$$

Hence, combining (3.1), 3.5), (3.6) and (3.7), we have

$$
\|\omega w\|_{1, \Omega}^{2} \lesssim h_{\Omega_{0}}\|w\|_{1, \Omega_{p}}^{2}+\|w\|_{0, \Omega_{p}}^{2}+\|f\|_{-1, \Omega_{0}}\left(h_{\Omega_{0}}\|w\|_{1, \Omega_{p}}+\|\omega w\|_{1, \Omega}\right),
$$

or

$$
\|w\|_{1, D} \lesssim h_{\Omega_{0}}^{1 / 2}\|w\|_{1, \Omega_{p}}+\|w\|_{0, \Omega_{0}}+\|f\|_{-1, \Omega_{0}} .
$$


The argument may be repeated for $\|w\|_{1, \Omega_{p}}$ on the right to yield

$$
\|w\|_{1, \Omega_{j}} \lesssim h_{\Omega_{0}}^{1 / 2}\|w\|_{1, \Omega_{j-1}}+\|w\|_{0, \Omega_{0}}+\|f\|_{-1, \Omega_{0}}, \quad j=1,2, \cdots, p .
$$

Combining (3.8) and (3.9), we get from Assumptions A.0 and A.2

$$
\begin{aligned}
\|w\|_{1, D} & \lesssim h_{\Omega_{0}}^{(p+1) / 2}\|w\|_{1, \Omega_{0}}+\|w\|_{0, \Omega_{0}}+\|f\|_{-1, \Omega_{0}} \\
& \lesssim h_{\Omega}^{(p+1) / 2}\left\|h^{-1} w\right\|_{0, \Omega_{0}}+\|w\|_{0, \Omega_{0}}+\|f\|_{-1, \Omega_{0}} \\
& \lesssim\|w\|_{0, \Omega_{0}}+\|f\|_{-1, \Omega_{0}} .
\end{aligned}
$$

This completes the proof.

The local stability of the Galerkin-projection is stated as follows.

Theorem 3.3. Let $u \in H_{0}^{1}(\Omega)$ and $D \subset \subset \Omega_{0}$. If Assumptions A.0, A.1, A.2 and A.3 hold, then

$$
\left\|P_{h} u\right\|_{1, D} \lesssim\|u\|_{1, \Omega_{0}}+\left\|P_{h} u\right\|_{0, \Omega_{0}} .
$$

Proof. Let $R_{h} \equiv P_{h}^{\Omega_{0}}: H_{0}^{1}\left(\Omega_{0}\right) \rightarrow S_{0}^{h}\left(\Omega_{0}\right)$ be the Galerkin projection, i.e., for $w \in H_{0}^{1}\left(\Omega_{0}\right)$,

$$
a\left(w-R_{h} w, v\right)=0, \quad \forall v \in S_{0}^{h}\left(\Omega_{0}\right) .
$$

Choose $D_{1} \subset \Omega$ satisfying $D \subset \subset D_{1} \subset \subset \Omega_{0}$ and $\omega \in C_{0}^{\infty}(\Omega)$ such that $\omega \equiv 1$ on $\bar{D}_{1}$ and $\operatorname{supp} \omega \subset \subset \Omega_{0}$. Then for $\tilde{u}=\omega u$,

$$
a\left(R_{h} \tilde{u}-P_{h} u, v\right)=0, \quad \forall v \in S_{h}^{0}\left(D_{1}\right) .
$$

Thus, Lemma 3.2 yields

$$
\left\|R_{h} \tilde{u}-P_{h} u\right\|_{1, D} \lesssim\left\|R_{h} \tilde{u}-P_{h} u\right\|_{0, D_{1}} .
$$

Therefore, estimates similar to (2.17) lead to

$$
\begin{aligned}
\left\|P_{h} u\right\|_{1, D} & \leq\left\|R_{h} \tilde{u}\right\|_{1, D}+\left\|R_{h} \tilde{u}-P_{h} u\right\|_{1, D} \\
& \lesssim\left\|R_{h} \tilde{u}\right\|_{1, D}+\left\|R_{h} \tilde{u}-P_{h} u\right\|_{0, D_{1}} \\
& \lesssim\left\|R_{h} \tilde{u}\right\|_{1, \Omega_{0}}+\left\|P_{h} u\right\|_{0, D_{1}} \\
& \lesssim\|\tilde{u}\|_{1, \Omega_{0}}+\left\|P_{h} u\right\|_{0, \Omega_{0}} \\
& \lesssim\|u\|_{1, \Omega_{0}}+\left\|P_{h} u\right\|_{0, \Omega_{0}} .
\end{aligned}
$$

Thus, we obtain (3.11). This completes the proof.

Theorem 3.4. Let $u \in H_{0}^{1}(\Omega)$ and $D \subset \subset \Omega_{0}$. If Assumptions A.0, A.1, A.2 and A.3 hold, then

$$
\left\|u-P_{h} u\right\|_{1, D} \lesssim \inf _{v \in S_{0}^{h}(\Omega)}\|u-v\|_{1, \Omega_{0}}+\left\|u-P_{h} u\right\|_{0, \Omega},
$$

or

$$
\left\|u-P_{h} u\right\|_{1, D} \lesssim \inf _{v \in S_{0}^{h}(\Omega)}\|u-v\|_{1, \Omega_{0}}+\rho_{\Omega}(h)\left\|u-P_{h} u\right\|_{1, \Omega} .
$$

Proof. Note that for any $v \in S_{0}^{h}(\Omega), P_{h} v=v$, we get from Theorem 3.3,

$$
\left\|P_{h} u-v\right\|_{1, D} \lesssim\|u-v\|_{1, \Omega_{0}}+\left\|P_{h} u-v\right\|_{0, \Omega_{0}}, \quad \forall v \in S_{0}^{h}(\Omega),
$$

which leads to (3.15). And (3.16) is derived from (3.15) and Lemma 2.1 This completes the proof. 
Corollary 3.5. Let $u \in H_{0}^{1}(\Omega), D \subset \subset \Omega_{0}$ and $0 \leq s \leq r$. If Assumptions A.0, A.1', A.2 and A.3 hold, then

$$
\left\|u-P_{h} u\right\|_{1, D} \lesssim h_{\Omega_{0}}^{s}\|u\|_{s+1, \Omega_{0}}+h_{\Omega}^{s+\alpha}\|u\|_{1+s, \Omega} .
$$

Remark 3.6. The results above show that many refined finite element meshes can be locally employed.

Remark 3.7. Similar results hold for $P_{h}^{*}$.

3.2. Local a posteriori error estimates. In this section, we shall present local a posteriori error estimate in energy-norm. First, we need the following technical result.

Lemma 3.8. Let $D \subset \subset \Omega_{0}$, and let $\omega \in C_{0}^{\infty}(\Omega)$ be such that supp $\omega \subset \subset \Omega_{0}$. Then

$$
a(\omega w, \phi) \leq a(w, \omega \phi)+C\|w\|_{0, \Omega_{0}}\|\phi\|_{1, \Omega_{0}}, \forall w, \phi \in H_{0}^{1}(\Omega) .
$$

Proof. From the identity

$a(\omega w, \phi)=a(w, \omega \phi)+\int_{\Omega}\left(\sum_{i, j=1}^{d}\left(a_{i j} \frac{\partial \omega}{\partial x_{i}} \frac{\partial \phi}{\partial x_{j}}+\frac{\partial}{\partial x_{i}}\left(a_{i j} \frac{\partial \omega}{\partial x_{j}} \phi\right)\right)+\sum_{i=1}^{d} b_{i} \frac{\partial \omega}{\partial x_{i}} \phi\right) w$,

we immediately obtain (3.18).

To state the a posteriori estimates, we need some more notation. Let $\partial T^{h}(\Omega)$ be the set of all the interior faces of the mesh $T^{h}(\Omega)$, and $\partial T^{h}(\tau)=\left\{F \in \partial T^{h}(\Omega)\right.$ : $F \subset \bar{\tau}$ \}. For each $F \in \partial T^{h}(\Omega)$, let $\mathbf{n}_{F}$ be a unit vector normal to $F$, and define for $v \in S^{h}(\Omega)$

$$
J_{F}(v)=\max _{x \in F}\left|\lim _{s \rightarrow 0^{+}} \mathbf{n}_{F}^{T}\left((A \nabla v)\left(x+s \mathbf{n}_{F}\right)-(A \nabla v)\left(x-s \mathbf{n}_{F}\right)\right)\right| .
$$

Namely, $J_{F}(v)$ is the jump across $F$ in the normal component of $A \nabla v$. We now introduce $\eta(v)$ by

$$
(\eta(v))(x)=|f(x)-(L v)(x)|+h^{-1} \max _{F \in \partial T^{h}(\tau)} J_{F}(v), \quad x \in \tau .
$$

One sees that $\eta\left(u_{h}\right), h \eta\left(u_{h}\right)$ and $h^{2} \eta\left(u_{h}\right)$ are computable in terms of the finite element solution $u_{h}\left(\equiv P_{h} u\right)$.

Theorem 3.9. Let $u \in H_{0}^{1}(\Omega)$ and $D \subset \subset \Omega_{0}$. If Assumptions A.1, A.3 and A.4 hold, then

$$
\left\|u-u_{h}\right\|_{1, D} \lesssim\left\|h \eta\left(u_{h}\right)\right\|_{0, \Omega_{0}}+\left\|u-u_{h}\right\|_{0, \Omega}
$$

or

$$
\left\|u-u_{h}\right\|_{1, D} \lesssim\left\|h \eta\left(u_{h}\right)\right\|_{0, \Omega_{0}}+\rho_{\Omega}(h)\left\|u-u_{h}\right\|_{1, \Omega} .
$$

Proof. Choose $D_{1} \subset \Omega$ satisfying $D \subset \subset D_{1} \subset \subset \Omega_{0}$ and $\omega \in C_{0}^{\infty}(\Omega)$ such that $\omega \equiv 1$ on $\bar{D}_{1}$ and $\operatorname{supp} \omega \subset \subset \Omega_{0}$. Thus, from Lemma 3.8, we have

$$
a(\omega e, \phi) \leq a\left(u-u_{h}, \omega \phi\right)+C\|e\|_{0, \Omega}\|\phi\|_{1, \Omega}, \quad \forall \phi \in H_{0}^{1}(\Omega),
$$

where $e=u-u_{h}$.

Note that for any $v \in S_{h}^{0}\left(\Omega_{0}\right)$,

$$
\begin{aligned}
a\left(u-u_{h}, \omega \phi\right) & =a\left(u-u_{h}, \omega \phi-v\right) \\
& =\sum_{\tau \in T^{h}\left(\Omega_{0}\right)}\left(\int_{\tau}\left(f-L u_{h}\right)(\omega \phi-v)-\sum_{F \in \partial T^{h}(\tau)} \int_{F} \mathbf{n}_{F}^{T} A \nabla u_{h}(\omega \phi-v)\right),
\end{aligned}
$$


and for any $F \in \partial T^{h}(\tau)$, there exists $\tilde{\tau} \in T^{h}(\Omega)$ such that $F \in \partial T^{h}(\tilde{\tau})$. Thus Assumption A.4 implies that

$$
\begin{aligned}
\mid & \sum_{\tau \in T^{h}\left(\Omega_{0}\right)} \sum_{F \in \partial T^{h}(\tau)} \int_{F} \mathbf{n}_{F}^{T} A \nabla u_{h}(\omega \phi-v) \mid \\
\leq & \frac{1}{2} \sum_{\tau \in T^{h}\left(\Omega_{0}\right)} \sum_{F \in \partial T^{h}(\tau)} \int_{F} J_{F}\left(u_{h}\right)|\omega \phi-v| \\
& \lesssim \sum_{\tau \in T^{h}\left(\Omega_{0}\right)} h_{\tau}^{(N-1) / 2} \max _{F \in \partial T^{h}(\tau)} J_{F}\left(u_{h}\right)\|\omega \phi-v\|_{0, F} \\
& \lesssim \sum_{\tau \in T^{h}\left(\Omega_{0}\right)} h_{\tau}^{N / 2} \max _{F \in \partial T^{h}(\tau)} J_{F}\left(u_{h}\right)\left(\left\|h^{-1}(\omega \phi-v)\right\|_{0, \tau}+\|\omega \phi-v\|_{1, \tau}\right) .
\end{aligned}
$$

Thus, we get, for any $v \in S_{h}^{0}\left(\Omega_{0}\right)$,

$$
\begin{aligned}
& \left|a\left(u-u_{h}, \omega \phi\right)\right| \\
& \quad \lesssim\left\|h \eta\left(u_{h}\right)\right\|_{0, \Omega_{0}}\left(\left\|h^{-1}(\omega \phi-v)\right\|_{0, \Omega_{0}}+\|\omega \phi-v\|_{1, \Omega_{0}}\right),
\end{aligned}
$$

which together with Assumptions A.1 and A.3 yields

$$
\left|a\left(u-u_{h}, \omega \phi\right)\right| \lesssim\left\|h \eta\left(u_{h}\right)\right\|_{0, \Omega_{0}}\|\phi\|_{1, \Omega_{0}} .
$$

Therefore, (2.13) and (3.22) lead to

$$
\|\omega e\|_{1, \Omega_{0}} \lesssim\left\|h \eta\left(u_{h}\right)\right\|_{0, \Omega_{0}}+\|e\|_{0, \Omega},
$$

which implies (3.20).

Remark 3.10. In (3.20) or (3.21), the last term is of higher order and hence negligible. If, for example, (2.15) holds with $\alpha=1$, one has the following a posterior error estimates, see e.g. [26, 28]:

$$
\left\|u-u_{h}\right\|_{0, \Omega} \leq\left\|h^{2} \eta\left(u_{h}\right)\right\|_{0, \Omega}
$$

under additional assumptions. Moreover,

$$
\left\|h^{2} \eta\left(u_{h}\right)\right\|_{0, \Omega}, \quad\left\|h \eta\left(u_{h}\right)\right\|_{0, \Omega}
$$

are globally equivalent to the errors $\left\|u-u_{h}\right\|_{0, \Omega}$ and $\left\|u-u_{h}\right\|_{1, \Omega}$, respectively, cf. e.g. [4, 26, 27, 28, 36, 44, 45] and reference cited therein. Similar arguments show that $\left\|h \eta\left(u_{h}\right)\right\|_{0, \Omega_{0}}$ is essentially controlled by $\left\|u-u_{h}\right\|_{1, \Omega_{0}}$. This means that we essentially have

$$
\left\|u-u_{h}\right\|_{1, D} \approx C\left\|h \eta\left(u_{h}\right)\right\|_{0, \Omega_{0}} .
$$

Remark 3.11. The argument here easily extends to other boundary conditions, provided the problems are well-posed. 


\section{NEW LOCAL AND PARALLEL ALGORITHMS}

In this section we shall present some new local and parallel finite element algorithms. These algorithms are motivated by the local error estimates studied in the previous section. We shall first discuss the local algorithms. The generalization of the local algorithms to parallel algorithms is straightforward.

For clarity, let $\Omega$ be a polygonal domain and $S_{0}^{h}(\Omega) \subset H_{0}^{1}(\Omega)$ be a finite element subspace of degree $r$ associated with a grid $T^{h}(\Omega)$. Let $u_{h} \in S_{0}^{h}(\Omega)$ be the solution of the standard finite element scheme for solving (2.11):

$$
a\left(u_{h}, v\right)=(f, v), \forall v \in S_{0}^{h}(\Omega) .
$$

Either locally or globally, with proper regularity assumption, we have the following error estimate:

$$
\left\|u-u_{h}\right\|_{1} \lesssim h^{s}, \quad 1 \leq s \leq r .
$$

With this type of error estimates in mind, in the rest of this section, we will only compare the approximate solutions from our new algorithms with $u_{h}$ instead of the exact solution $u$.

4.1. Local algorithms. The local algorithms we shall now present can be used to obtain approximate solution on a given subdomain, mostly by local computation. The main idea is that the more global component of a finite element solution may be obtained by a relatively coarser grid, and the rest of the computation can then be localized.

Roughly speaking, our new algorithms will be based on sometimes one coarse grid of size $H$ and one fine grid of size $h \ll H$, and sometimes on a grid that is fine in a subdomain and coarse on the rest of the domain. The fine grid may be only defined locally. In our analysis, we shall use an auxiliary fine grid, say $T^{h}(\Omega)$, that is globally defined. One basic assumption for this auxiliary fine grid is that it should coincide with the local fine grid in the subdomain of interest.

Let $T^{H}(\Omega)$ be a shape-regular coarse grid, of size $H \gg h$, so that the highly locally refined mesh $T^{h}\left(\Omega_{0}\right)$ can be obtained, where $\Omega_{0}$ is a slightly larger subdomain containing a subdomain $D \subset \Omega$ (namely $D \subset \subset \Omega_{0}$ ), see Figure 3. More precisely, we let $T_{H}^{h}(\Omega)$ denote a locally refined shape-regular mesh that may be viewed as being obtained by refining $T^{H}(\Omega)$ locally around the subdomain $D$ in such a way that $T_{H}^{h}\left(\Omega_{0}\right)=T^{h}\left(\Omega_{0}\right)$. We are interested in obtaining the approximation solution in the given subdomain $D$ with an accuracy comparable to that from $T^{h}(\Omega)$. We shall propose two different gridding strategies for obtaining finite element approximations on the subdomain $D$ (see Figure 4). We denote the corresponding finite element space by $S_{0}^{H, h}(\Omega) \subset H_{0}^{1}(\Omega)$, which consists of piecewise polynomial of degree less than or equal to $r$ in this section.

4.1.1. First approach. The first strategy is simply to solve a standard finite element solution in $S_{0}^{H, h}(\Omega)$.

Algorithm A0. Find $u_{H}^{h} \in S_{0}^{H, h}(\Omega)$ such that

$$
a\left(u_{H}^{h}, v\right)=(f, v), \quad \forall v \in S_{0}^{H, h}(\Omega) .
$$

Strictly speaking, this algorithm is still a global algorithm as a global problem is solved. But it is designed to obtain a local approximation in the subdomain $D$ and it makes use of a mesh that is much coarser away from $D$. 

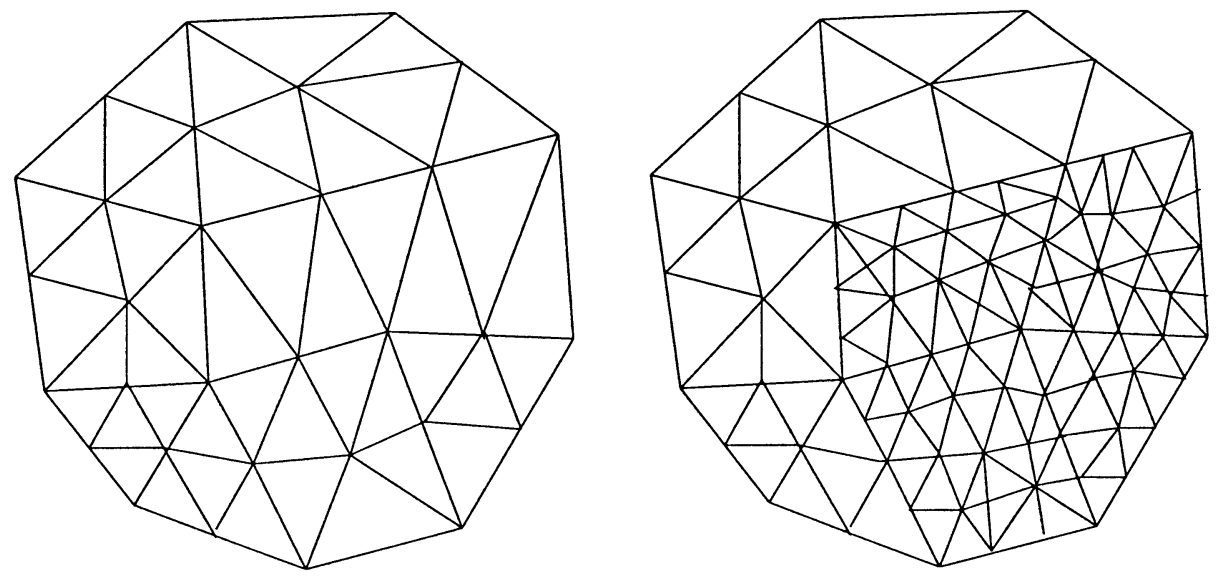

FiguRE 3. Local Refinement
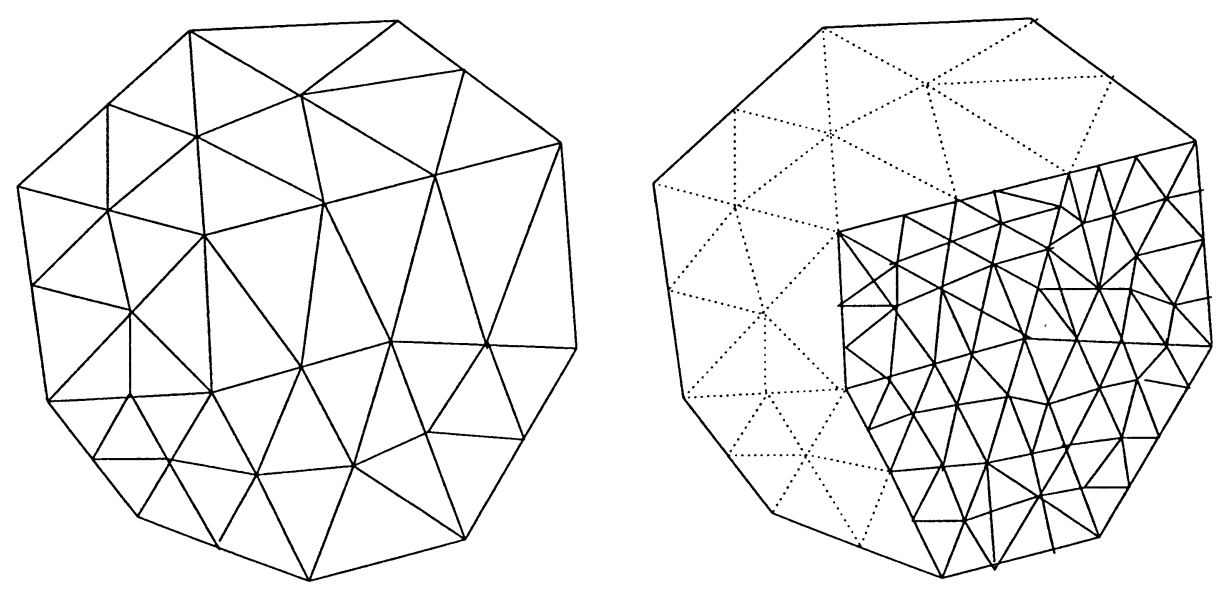

FIGURE 4. Localization

Theorem 4.1. Assume that $u_{H}^{h} \in S_{0}^{H, h}(\Omega)$ is obtained by Algorithm A0. Then

$$
\left\|u_{h}-u_{H}^{h}\right\|_{1, D} \lesssim\left\|u_{h}-u_{H}^{h}\right\|_{0, \Omega} \lesssim H^{r+\alpha}|u|_{r+1, \Omega} .
$$

Proof. By the definition of Algorithm A0 and our assumption on the auxiliary grid $T^{h}(\Omega)$ that coincides with $T_{H}^{h}(\Omega)$ on $\Omega_{0}$, we have

$$
a\left(u_{H}^{h}-u_{h}, v\right)=0, \quad \forall v \in S_{h}^{0}\left(\Omega_{0}\right) .
$$

By Lemma 3.2, we get

$$
\left\|u_{h}-u_{H}^{h}\right\|_{1, D} \lesssim\left\|u_{h}-u_{H}^{h}\right\|_{0, \Omega_{0}}
$$

and then finish the proof.

We would like to remark here that similar locally refined grids have been used for different purposes in the literature, cf. Bramble [19], Bramble, Ewing, Parashkevov and Pasciak [20], and Bramble, Ewing, Pasciak and Schatz [21]. 
4.1.2. Second approach. Our second strategy is in a way an improvement of the first strategy. In this strategy, we first solve a global problem only on the given coarse grid $T^{H}(\Omega)$, and we then correct the residue locally on the fine mesh $T^{h}\left(\Omega_{0}\right)$ (= $\left.T_{H}^{h}\left(\Omega_{0}\right)\right)$. Let $S_{0}^{H}(\Omega) \subset H_{0}^{1}(\Omega)$ be the finite element space of degree $r$ defined on $T^{H}(\Omega)$.

A prototype of our new local algorithms is as follows.

Algorithm B0. 1. Find a global coarse grid solution $u_{H} \in S_{0}^{H}(\Omega)$ :

$$
a\left(u_{H}, v\right)=(f, v), \quad \forall v \in S_{0}^{H}(\Omega) .
$$

2. Find a local fine grid correction $e_{h} \in S_{0}^{h}\left(\Omega_{0}\right)$ :

$$
a\left(e_{h}, v\right)=(f, v)-a\left(u_{H}, v\right), \quad \forall v \in S_{0}^{h}\left(\Omega_{0}\right) .
$$

3. Update: $u^{h}=u_{H}+e_{h}$, in $\Omega_{0}$.

Theorem 4.2. Assume that $u^{h} \in S^{h}\left(\Omega_{0}\right)$ is obtained by Algorithm B0, and Assumption $R\left(\Omega_{0}\right)$ holds. Then

$$
\left\|u_{h}-u^{h}\right\|_{1, D} \lesssim\left\|u_{h}-u_{H}\right\|_{0, \Omega}+H^{\alpha}\left\|u_{h}-u_{H}\right\|_{1, \Omega} \lesssim H^{r+\alpha}|u|_{r+1, \Omega} .
$$

Proof. By the definition of Algorithm B0,

$$
a\left(u^{h}-u_{h}, v\right)=0, \quad \forall v \in S_{h}^{0}\left(\Omega_{0}\right) .
$$

By Lemma 3.2, we get

$$
\left\|u_{h}-u^{h}\right\|_{1, D} \lesssim\left\|u^{h}-u_{h}\right\|_{0, \Omega_{0}} \lesssim\left\|u_{h}-u_{H}\right\|_{0, \Omega_{0}}+\left\|e_{h}\right\|_{0, \Omega_{0}} .
$$

To estimate $\left\|e_{h}\right\|_{0, \Omega_{0}}$, we use the Aubin-Nitsche duality argument. Given any $\phi \in$ $L^{2}\left(\Omega_{0}\right)$, there exists $w \in H_{0}^{1}\left(\Omega_{0}\right)$ such that

$$
a(v, w)=(\phi, v), \quad \forall v \in H_{0}^{1}\left(\Omega_{0}\right) .
$$

Let $w_{h}^{0} \in S_{0}^{h}\left(\Omega_{0}\right)$ and $w_{H}^{0} \in S_{0}^{H}\left(\Omega_{0}\right)$ satisfy

$$
a\left(v_{h}, w_{h}^{0}\right)=a\left(v_{h}, w\right), \quad \forall v_{h} \in S_{0}^{h}\left(\Omega_{0}\right), \quad a\left(v_{H}, w_{H}^{0}\right)=a\left(v_{H}, w\right), \quad \forall v_{H} \in S_{0}^{H}\left(\Omega_{0}\right) .
$$

Then

$$
\left\|w-w_{h}^{0}\right\|_{1, \Omega_{0}} \lesssim h^{\alpha}\|\phi\|_{0, \Omega_{0}}, \quad\left\|w-w_{H}^{0}\right\|_{1, \Omega} \lesssim H^{\alpha}\|\phi\|_{0, \Omega_{0}} .
$$

It follows that

$$
\begin{aligned}
\left(e_{h}, \phi\right) & =a\left(e_{h}, w\right)=a\left(e_{h}, w_{h}^{0}\right)=a\left(u_{h}-u_{H}, w_{h}^{0}\right) \\
& =a\left(u_{h}-u_{H}, w_{h}^{0}-w\right)+a\left(u_{h}-u_{H}, w\right) \\
& =a\left(u_{h}-u_{H}, w_{h}^{0}-w\right)+a\left(u_{h}-u_{H}, w-w_{H}^{0}\right) \\
& \lesssim H^{\alpha}\left\|u_{h}-u_{H}\right\|_{1, \Omega}\|\phi\|_{0, \Omega_{0}}
\end{aligned}
$$

which implies

$$
\left\|e_{h}\right\|_{0, \Omega_{0}} \lesssim H^{\alpha}\left\|u_{h}-u_{H}\right\|_{1, \Omega} .
$$

The desired result then follows.

Following the basic idea in Xu [48, 50, 51, for non-SPD problems Algorithm B0 may be modified in such a way that the local fine grid correction in Algorithm B0 is only carried out for the symmetric positive definite leading part of the equation.

Algorithm C0. 1. Find a global coarse grid solution $u_{H} \in S_{0}^{H}(\Omega)$ :

$$
a\left(u_{H}, v\right)=(f, v), \quad \forall v \in S_{0}^{H}(\Omega) .
$$


2. Find a local fine grid correction $e_{h} \in S_{0}^{h}\left(\Omega_{0}\right)$ :

$$
a_{0}\left(e_{h}, v\right)=(f, v)-a\left(u_{H}, v\right), \quad \forall v \in S_{0}^{h}\left(\Omega_{0}\right) .
$$

3. Update: $u^{h}=u_{H}+e_{h}$, in $\Omega_{0}$.

Theorem 4.3. Assume that $u^{h} \in S^{h}\left(\Omega_{0}\right)$ is obtained by Algorithm C0. Then

$$
\left\|u_{h}-u^{h}\right\|_{1, D} \lesssim\left\|u_{h}-u_{H}\right\|_{0, \Omega}+H^{\alpha}\left\|u_{h}-u_{H}\right\|_{1, \Omega} \lesssim H^{r+\alpha}|u|_{r+1, \Omega} .
$$

Proof. By the definition of Algorithm C0,

$$
a_{0}\left(u^{h}-u_{h}, v\right)=N\left(u_{h}-u_{H}, v\right), \quad \forall v \in S_{h}^{0}\left(\Omega_{0}\right) .
$$

Thus, by Lemma 3.2 , we obtain

$$
\left\|u^{h}-u_{h}\right\|_{1, D} \lesssim\left\|u^{h}-u_{h}\right\|_{0, \Omega_{0}}+\left\|u_{h}-u_{H}\right\|_{0, \Omega_{0}} \lesssim\left\|u_{h}-u_{H}\right\|_{0, \Omega_{0}}+\left\|e_{h}\right\|_{0, \Omega_{0}} .
$$

The rest of the proof is similar to that of Theorem 4.2, and we leave the details to the interested readers.

4.2. New parallel algorithms based on local algorithms. The parallel algorithms we shall present here are naturally obtained from the local algorithms that we studied above. Given an initial coarse triangulation $T^{H}(\Omega)$, let us first divide $\Omega$ into a number of disjoint subdomains $D_{1}, \ldots, D_{m}$ (see Figure 5), then enlarge each $D_{j}$ to obtain $\Omega_{j}$ that align with $T^{H}(\Omega)$. The basic idea of our parallel algorithm is very simple: we just apply the local algorithms in parallel in all $\Omega_{j}$ 's.

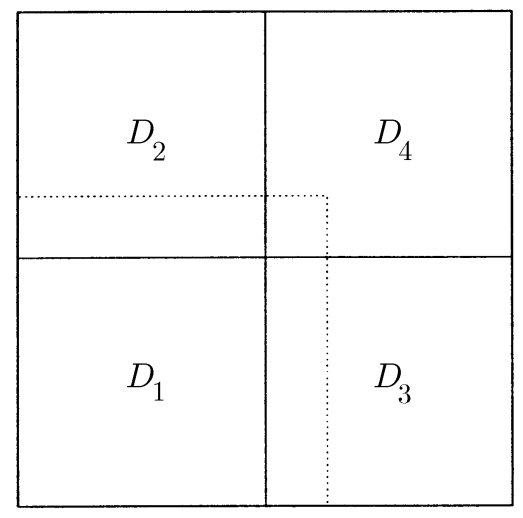

FiguRE 5. Domain decomposition: $D_{j} \subset \subset \Omega_{j}$

4.2.1. Basic parallel algorithms. Let us first discuss the parallel version of Algorithm A0. For each $j$, we use some adaptive process to obtain a shape-regular mesh $T_{j}(\Omega)$ and the corresponding finite element solution denoted by $u_{j}$. We note that each $T_{j}(\Omega)$ looks like the mesh depicted in Figure 1 and it has a substantially finer mesh inside $\Omega_{j}$. We note that all $T_{j}(\Omega)$ are different triangulations for $\Omega$ and they can be very arbitrary; but for simplicity of exposition, we assume each $T_{j}(\Omega)$ has the same size $h$ in $\Omega_{j}$ (more precisely, $T_{j}\left(\Omega_{j}\right)=T^{h}\left(\Omega_{j}\right)$ ) and has the size $H$ away from $\Omega_{j}$. Let $S_{0}^{h_{j}}(\Omega) \subset H_{0}^{1}(\Omega)$ be the corresponding finite element spaces.

Algorithm A1. 1. Find $u_{j} \in S_{0}^{h_{j}}(\Omega)(j=1, \ldots, m)$ in parallel:

$$
a\left(u_{j}, v\right)=(f, v), \quad \forall v \in S_{0}^{h_{j}}(\Omega) .
$$


2. Set $u^{h}=u_{j}$, in $D_{j}(j=1, \ldots, m)$.

Define a piecewise norm

$$
\left\|u_{h}-u^{h}\right\|_{1, \Omega}=\left(\sum_{j=1}^{m}\left\|u_{h}-u^{h}\right\|_{1, D_{j}}^{2}\right)^{1 / 2} .
$$

By Theorem 4.1, we have

$$
\left\|u_{h}-u^{h}\right\|_{1, \Omega} \lesssim H^{r+\alpha}|u|_{r+1, \Omega} .
$$

Consequently,

$$
\left\|u-u^{h}\right\|_{1, \Omega} \lesssim\left(h^{r}+H^{r+\alpha}\right)|u|_{r+1, \Omega} .
$$

We now discuss the parallel versions of Algorithms B0 and C0. Although there are many possibilities for the generalization, for clarity of exposition, it appears to be most convenient to discuss these using two globally defined grids: an initial coarse grid $T^{H}(\Omega)$ and a refined (from $T^{H}(\Omega)$ ) grid $T^{h}(\Omega)$ that satisfies $h \ll H$.

Algorithm B1. 1. Find a global coarse grid solution $u_{H} \in S_{0}^{H}(\Omega)$ :

$$
a\left(u_{H}, v\right)=(f, v), \quad \forall v \in S_{0}^{H}(\Omega) .
$$

2. Find local fine grid corrections $e_{h}^{j} \in S_{0}^{h}\left(\Omega_{j}\right)(j=1, \ldots, m)$ in parallel:

$$
a\left(e_{h}^{j}, v\right)=(f, v)-a\left(u_{H}, v\right), \quad \forall v \in S_{0}^{h}\left(\Omega_{j}\right),
$$

3. Set $u^{h}=u_{H}+e_{h}^{j}$, in $D_{j}(j=1, \ldots, m)$.

By Theorem 4.2, for this algorithm, we apparently have the following result.

Theorem 4.4. Assume that $u^{h}$ is the solution obtained by Algorithm B1 and Assumptions $R\left(\Omega_{j}\right)$ hold for $j=1,2, \cdots, m$. Then

$$
\left\|u_{h}-u^{h}\right\|_{1, \Omega} \lesssim H^{\alpha}\left\|u_{h}-u_{H}\right\|_{1, \Omega} \lesssim H^{r+\alpha}|u|_{r+1, \Omega}
$$

and

$$
\left\|u-u^{h}\right\|_{1, \Omega} \lesssim\left(h^{r}+H^{r+\alpha}\right)|u|_{r+1, \Omega} .
$$

Proof. Note that

$$
\left\|u_{h}-u_{H}\right\|_{0, \Omega} \lesssim H^{\alpha}\left\|u_{h}-u_{H}\right\|_{1, \Omega} .
$$

The desired result follows.

Algorithm C1. 1. Find a global coarse grid solution $u_{H} \in S_{0}^{H}(\Omega)$ :

$$
a\left(u_{H}, v\right)=(f, v), \quad \forall v \in S_{0}^{H}(\Omega) .
$$

2. Find local fine grid corrections $e_{h}^{j} \in S_{0}^{h}\left(\Omega_{j}\right)(j=1, \ldots, m)$ in parallel:

$$
a_{0}\left(e_{h}^{j}, v\right)=(f, v)-a\left(u_{H}, v\right), \quad \forall v \in S_{0}^{h}\left(\Omega_{j}\right) .
$$

3. Update: $u^{h}=u_{H}+e_{h}^{j}$, in $D_{j}(j=1, \ldots, m)$.

For this algorithm, we have

Theorem 4.5. Assume that $u^{h}$ is the solution obtained by Algorithm C1. Then

$$
\left\|u_{h}-u^{h}\right\|_{1, \Omega} \lesssim H^{\alpha}\left\|u_{h}-u_{H}\right\|_{1, \Omega} \lesssim H^{r+\alpha}|u|_{r+1, \Omega}
$$

and

$$
\left\|u-u^{h}\right\|_{1, \Omega} \lesssim\left(h^{r}+H^{r+\alpha}\right)|u|_{r+1, \Omega}
$$


4.2.2. Further modifications. We note that the approximations $u^{h}$ obtained by Algorithms A1, B1 and C1 are piecewise defined and they are in general discontinuous. We also point out that $\left\|u_{h}-u^{h}\right\|_{0, \Omega}$ does not in general have higher order than $\left\|u_{h}-u^{h}\right\|_{1, \Omega}$. In this subsection, we shall propose some further modifications for these algorithms to achieve the following two goals:

1. smooth $u^{h}$ to obtain a global $H^{1}(\Omega)$ approximation;

2. improve the error $\left\|u_{h}-u^{h}\right\|_{0, \Omega}$.

The first goal will be achieved by using some more local fine grid problems; the second will be achieved by carrying out a further global coarse grid correction. We note that the second goal is realized after the first goal has been achieved.

We now proceed to present a modified algorithm that addresses both of the aforementioned two issues. To do this, we pick another sequence of subdomains $G_{j} \subset \subset D_{j}$ and $G_{m+1}=\Omega \backslash\left(\bigcup_{j=1}^{m} \bar{G}_{j}\right)$ (see Figure 6 ).

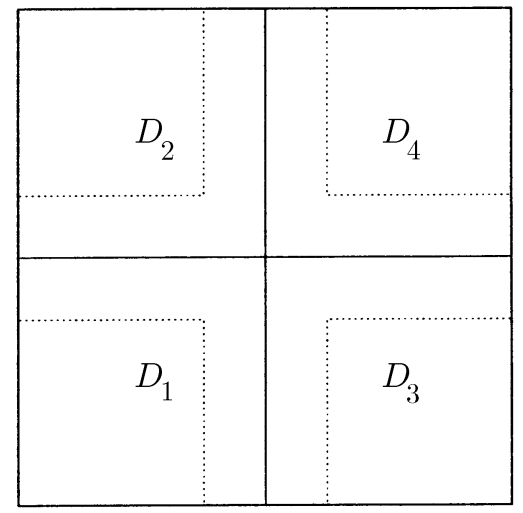

Figure 6. Domain decomposition: $G_{j} \subset \subset D_{j} \subset \subset \Omega_{j}$ and $G_{m+1}$

Algorithm C2. 1. Find a global coarse grid solution $u_{H} \in S_{0}^{H}(\Omega)$ :

$$
a\left(u_{H}, v\right)=(f, v), \quad \forall v \in S_{0}^{H}(\Omega) .
$$

2. Find local fine grid corrections $e_{h}^{j} \in S_{0}^{h}\left(\Omega_{j}\right)(j=1, \ldots, m)$ in parallel:

$$
a_{0}\left(e_{h}^{j}, v\right)=(f, v)-a\left(u_{H}, v\right), \quad \forall v \in S_{0}^{h}\left(\Omega_{j}\right),
$$

3. Set $u^{h}=u_{H}+e_{h}^{j}$, in $G_{j}(j=1, \ldots, m)$ and $u^{h}$ on $\bar{G}_{m+1}$ is defined by $\left.u^{h}\right|_{\partial G_{j} \cap \partial G_{m+1}}=u_{H}+e_{h}^{j}(j=1, \ldots, m)$ and satisfying

$$
a_{0}\left(u^{h}, v\right)=(f, v)-N\left(u_{H}, v\right), \quad \forall v \in S_{0}^{h}\left(G_{m+1}\right) .
$$

4. Find a further coarse grid correction $e_{H} \in S_{0}^{H}(\Omega)$ :

$$
a\left(e_{H}, v\right)=(f, v)-a\left(u^{h}, v\right), \quad \forall v \in S_{0}^{H}(\Omega) .
$$

5. Update: $\tilde{u}^{h}=u^{h}+e_{H}$, in $\Omega$.

In the above algorithm, Step 3 is for obtaining a global $H^{1}$ solution and Step 4 is for improving the $L^{2}$ error.

For the analysis of the above algorithm, we need an additional assumption on the finite element space. 
Theorem 4.6. Assume that $u^{h}$ is the solution obtained by Algorithm C2 and Assumption A.5. Then

$$
\left\|u_{h}-u^{h}\right\|_{1, \Omega} \lesssim H^{\alpha}\left\|u_{h}-u_{H}\right\|_{1, \Omega} \lesssim H^{r+\alpha}|u|_{r+1, \Omega}
$$

and

$$
\left\|u_{h}-\tilde{u}^{h}\right\|_{0, \Omega} \lesssim H^{2 \alpha}\left\|u_{h}-u_{H}\right\|_{1, \Omega} \lesssim H^{r+2 \alpha}|u|_{r+1, \Omega} .
$$

Proof. From the definition, we have

$$
a_{0}\left(u_{h}-u^{h}, v\right)=N\left(u_{H}-u_{h}, v\right), \quad \forall v \in S_{0}^{h}\left(G_{m+1}\right) .
$$

Let

$$
a_{G_{m+1}}(u, v)=\int_{G_{m+1}} \sum_{i, j=1}^{d} a_{i j} \frac{\partial u}{\partial x_{i}} \frac{\partial v}{\partial x_{j}}
$$

then

$$
\left\|\nabla\left(u_{h}-u^{h}\right)\right\|_{0, G_{m+1}}^{2} \lesssim a_{G_{m+1}}\left(u_{h}-u^{h}, u_{h}-u^{h}\right) .
$$

Thus, for any $v \in S_{0}^{h}\left(G_{m+1}\right)$

$$
\left\|\nabla\left(u_{h}-u^{h}\right)\right\|_{0, G_{m+1}}^{2} \lesssim a_{G_{m+1}}\left(u_{h}-u^{h}, u_{h}-u^{h}-v\right)+\left\|u_{h}-u_{H}\right\|_{0, \Omega}\|v\|_{1, \Omega}
$$

and

$$
\begin{aligned}
&\left\|\nabla\left(u_{h}-u^{h}\right)\right\|_{0, G_{m+1}}^{2} \lesssim\left\|\nabla\left(u_{h}-u^{h}\right)\right\|_{0, G_{m+1}} \inf _{\chi \in S_{0}^{h}\left(G_{m+1}\right)}\left\|u_{h}-u^{h}-\chi\right\|_{1, G_{m+1}} \\
&+\left\|u_{h}-u_{H}\right\|_{0, \Omega} \inf _{\chi \in S_{0}^{h}\left(G_{m+1}\right)}\left(\left\|u_{h}-u^{h}-\chi\right\|_{1, G_{m+1}}+\|\chi\|_{1, \Omega}\right) .
\end{aligned}
$$

From Assumption A.5, we have

$$
\begin{aligned}
& \left\|\nabla\left(u_{h}-u^{h}\right)\right\|_{0, G_{m+1}}^{2} \lesssim\left\|\nabla\left(u_{h}-u^{h}\right)\right\|_{0, G_{m+1}}\left\|u_{h}-u^{h}\right\|_{1 / 2, \partial G_{m+1}} \\
& +\left\|u_{h}-u_{H}\right\|_{0, \Omega}\left(\left\|u_{h}-u^{h}\right\|_{1 / 2, \partial G_{m+1}}+\left\|u_{h}-u^{h}\right\|_{1, G_{m+1}}\right) .
\end{aligned}
$$

Using

$$
\left\|u_{h}-u^{h}\right\|_{1 / 2, \partial G_{m+1}} \lesssim \sum_{j=1}^{m}\left\|u_{h}-u^{h}\right\|_{1 / 2, \partial G_{j}} \lesssim \sum_{j=1}^{m}\left\|u_{h}-u^{h}\right\|_{1, G_{j}},
$$

or

$$
\left\|u_{h}-u^{h}\right\|_{1 / 2, \partial G_{m+1}} \lesssim\left\|u_{h}-u^{h}\right\|_{1, \Omega},
$$

we get

$$
\begin{aligned}
\left\|\nabla\left(u_{h}-u^{h}\right)\right\|_{0, G_{m+1}}^{2} & \lesssim\left\|u_{h}-u^{h}\right\|_{1, \Omega}^{2} \\
& +\left(\left\|u_{h}-u^{h}\right\|_{1, \Omega}+\left\|u_{h}-u^{h}\right\|_{1, G_{m+1}}\right)\left\|u_{h}-u_{H}\right\|_{0, \Omega} .
\end{aligned}
$$

Thus

$$
\begin{aligned}
& \left\|u_{h}-u^{h}\right\|_{1, \Omega}^{2} \lesssim\left\|\nabla\left(u_{h}-u^{h}\right)\right\|_{0, \Omega}^{2} \\
\lesssim & \left\|u_{h}-u^{h}\right\|_{1, \Omega}^{2}+\left\|u_{h}-u_{H}\right\|_{0, \Omega}^{2}+\left\|u_{h}-u^{h}\right\|_{1, \Omega}\left\|u_{h}-u_{H}\right\|_{0, \Omega},
\end{aligned}
$$

namely,

$$
\left\|u_{h}-u^{h}\right\|_{1, \Omega} \lesssim\left\|u_{h}-u^{h}\right\|_{1, \Omega}+\left\|u_{h}-u_{H}\right\|_{0, \Omega}
$$


which together with Theorem 4.5 and

$$
u_{h}-\tilde{u}^{h}=\left(I-P_{H}\right)\left(u_{h}-u^{h}\right)
$$

finishes the proof.

\section{LOCAL AND PARALLEL ADAPTIVE PROCESS}

The local error estimates and local/parallel algorithms presented in previous sections make it possible to design many new local and parallel adaptive algorithms for finite element computations. In this section, we shall give some examples.

5.1. On the traditional adaptive process. Before we present our new local and parallel adaptive method, we would like to recall some traditional finite element adaptive process based on a posteriori error estimates. We would like to illustrate that our local a posteriori error estimates give a certain theoretical justification of some traditional finite element adaptive techniques.

The basic idea of an adaptive algorithm is to use a given computed finite element solution to detect the behavior of the exact solution so that the underlying finite element meshes can get properly refined or de-refined in certain regions of the domain according to the behavior of the solution. The behavior of the solution is detected by using certain a posteriori error estimates like the ones presented in §3.2. In the existing literature, these a posteriori error estimates are often given and analyzed in a global form. For example, in view of (3.19), one can often use the following kind of global a posteriori error estimate:

$$
\left\|u-u_{h}\right\|_{1, \Omega} \leq E_{h} \equiv C_{0}\left\|h \eta\left(u_{h}\right)\right\|_{0, \Omega} .
$$

The constant $C_{0}$ only depends on the shape of the grids, and it may be properly estimated, or, for simplicity, one may take $C_{0}=1$. In practice, we wish to find a mesh $T^{h}(\Omega)$ (with least possible number of nodes) such that the corresponding finite element approximation $u_{h}$ satisfies

$$
\left\|u-u_{h}\right\|_{1, \Omega} \leq \delta
$$

for a given a tolerance error $\delta$.

Using, for example, (5.1), it suffices to refine the mesh in such a way that

$$
E_{h} \equiv C_{0}\left\|h \eta\left(u_{h}\right)\right\|_{0, \Omega} \leq \delta .
$$

If the above estimate is satisfied for the given mesh, then we have achieved our goal. Otherwise, we need to further refine the mesh locally. In order to use the global error estimate for local mesh refinement, one often uses the principle of equi-distribution for the error. Let $N_{h}$ be the total number of elements. For each element, we then check if the following is satisfied:

$$
C_{0}^{2}\left\|h \eta\left(u_{h}\right)\right\|_{0, \tau} \leq \delta^{2} / N_{h} .
$$

Here $\delta^{2} / N_{h}$ is the averaged error on $\tau$ obtained by the aforementioned equidistribution principle.

One natural question to ask is why a global a posteriori error estimate like (5.1) can be used locally as in (5.3). We would like to argue that our local estimates would give a theoretical justification of the aforementioned local application of a posteriori error estimates. One argument we can make is that the a posteriori error estimate itself is essentially local, according to Theorem [3.9. Another related 
argument can be based on the locality of a priori error estimates for $u-u_{h}$ according to Theorem 3.4

5.2. A local adaptive process. The locality of both a priori and a posteriori error estimates can be used to devise some local/parallel adaptive algorithms. As an example, we shall now propose a local adaptive algorithm that can be used to obtain an approximate solution that has a desired accuracy in a given subdomain locally.

Let $D \subset \Omega$ be a given subdomain and $\delta$ a given tolerance. Suppose we want to obtain an approximate solution $u_{h}$ satisfying

$$
\left\|u-u_{h}\right\|_{1, D} \leq \delta .
$$

According to the local error estimates in previous sections, this error tolerance can be achieved by only local mesh refinement around the domain $D$. Let $\Omega_{0}$ be a subdomain that is slightly larger than $D$. By Theorem 3.9 we have the estimate

$$
\left\|u-u_{h}\right\|_{1, D} \leq C_{0}\left\|h \eta\left(u_{h}\right)\right\|_{0, \Omega_{0}}+\text { higher order global error term. }
$$

If our initial grid is reasonably fine, the higher order global error term is negligible. This means we may only need to refine the mesh in the subdomain $\Omega_{0}$ so that

$$
C_{0}\left\|h \eta\left(u_{h}\right)\right\|_{0, \Omega_{0}} \leq \delta .
$$

This adaptive process corresponds precisely to the local algorithm described in 4.1.1 where a priori error estimates are discussed.

In view of the local algorithm described in 84.1 .2 apparently, a corresponding local adaptive process can also be obtained. We omit the details here.

5.3. A parallel adaptive process. As before, a simultaneous application of a local algorithm on a number of subdomains naturally leads to a parallel algorithm. In this subsection, we shall give some details for a parallel adaptive algorithm corresponding to the local adaptive algorithm described in the previous subsection.

Our goal is to design a parallel procedure to find a finite element approximation $u_{h}$ (which may be piecewise defined) satisfying (see (4.1))

$$
\left\|u-u_{h}\right\|_{1, \Omega} \leq \delta
$$

for a given tolerance error $\delta$.

Based on a reasonable good initial mesh, denoted by $T^{H}(\Omega)$, and its corresponding solution, denoted by $u_{H}$, an adaptive process is to make use of some a posteriori estimates based on information from $T^{H}(\Omega)$ and $u_{0}$ to adaptively come up with better and better meshes until a desired error tolerance is reached. Traditionally, after a stage of refinement/de-refinement, the a posteriori estimates are evaluated on the whole domain. Thanks to the local estimate (3.21), we propose that a posteriori estimates can be evaluated concurrently on a number of proper subdomains and a parallel adaptive process can then be brought about.

As in 4.2 , given an initial coarse triangulation $T^{H}(\Omega)$, we divide $\Omega$ into a number of disjoint subdomains $D_{1}, \ldots, D_{m}$, then enlarge each $D_{j}$ to obtain $\Omega_{j}$ 's that align with $T^{H}(\Omega)$.

We aim to reach (5.5) by refining the mesh $T^{H}(\Omega)$. Note that $\left\|u-u_{H}\right\|_{0, \Omega}$ is of higher order compared with $\left\|u-u_{H}\right\|_{1, \Omega}$; for convenience of exposition, we may assume that our initial mesh is fine enough so that $\left\|u-u_{H}\right\|_{0, \Omega}$ is much smaller than $\delta$. (This assumption is not crucial in practice, as $T^{H}(\Omega)$ can get updated by 
finer meshes in an adaptive process.) Thanks to the estimate (3.21), we have (with $h=H)$

$$
\left\|u-u_{h}\right\|_{1, D_{j}} \approx C_{j}\left\|h \eta\left(u_{h}\right)\right\|_{0, \Omega_{j}} .
$$

We use a standard principle of equi-distribution for the error control, in which we equalize the contribution from each subdomain. More precisely, the finite element approximation computed on the targeted mesh $T^{h}(\Omega)$ in terms of computational work satisfies

$$
\left\|u-u_{h}\right\|_{1, D_{j}}^{2} \leq \frac{\delta^{2}}{m}, \quad j=1,2, \cdots, m
$$

Therefore we can just carry out the mesh refinement locally on $\Omega_{j}$ until the following estimates are satisfied:

$$
C_{j}\left\|h \eta\left(u_{h}\right)\right\|_{0, \Omega_{j}}^{2} \leq \frac{\delta^{2}}{m}, \quad j=1,2, \cdots, m
$$

We note that the refinement process on each $\Omega_{j}$ is independent of those from other subdomains. Associated with each $\Omega_{j}$, we get a locally refined mesh as in Figure 1 and then find the corresponding finite element solution, denoted by $u_{j}$, on such a mesh. After all these are done, we then take final solutions that are defined piecewise on each $D_{j}$ restricted from $\Omega_{j}$.

The above exposition contains the main ideas of a parallel adaptive process, but for its application, there are many practical issues that need to be addressed. We refer to Bank and Holst [12] for a similar approach and implementation details.

\section{Some numerical EXPERIMENTS}

In this paper, we have presented many new estimates and new algorithms. It is perhaps a little too much of an undertaking to carry out and report numerical experiments for all these results in this single work. For illustration, we choose to report some simple numerical experiments only for Algorithms B1, C1 and C2.

We consider the simple unit square domain $\Omega=(0,1) \times(0,1)$ and a uniform triangulation $T^{h}(\Omega)=\{\tau\}$ (see Figure [7) and piecewise linear finite element spaces:

$S^{h}(\Omega)=\left\{v \in H^{1}(\Omega):\left.v\right|_{\tau}\right.$ is linear, $\left.\forall \tau \in T^{h}(\Omega)\right\}, \quad S_{0}^{h}(\Omega)=S^{h}(\Omega) \cap H_{0}^{1}(\Omega)$.

Divide $\Omega$ into four subdomains (see Figure 8 ):

$$
\begin{array}{ll}
D_{1}=(0,1 / 2) \times(0,1 / 2), & D_{2}=(0,1 / 2) \times(1 / 2,1), \\
D_{3}=(1 / 2,1) \times(0,1 / 2), & D_{4}=(1 / 2,1) \times(1 / 2,1) .
\end{array}
$$

Set

$$
\begin{array}{ll}
G_{1}=(0,3 / 8) \times(0,3 / 8), & G_{2}=(0,3 / 8) \times(5 / 8,1), \\
G_{3}=(5 / 8,1) \times(0,3 / 8), & G_{4}=(5 / 8,1) \times(5 / 8,1),
\end{array}
$$

and

$$
\begin{array}{ll}
\Omega_{1}=(0,5 / 8) \times(0,5 / 8), & \Omega_{2}=(0,5 / 8) \times(3 / 8,1), \\
\Omega_{3}=(3 / 8,1) \times(0,5 / 8), & \Omega_{4}=(3 / 8,1) \times(3 / 8,1) .
\end{array}
$$

Now we apply Algorithm B1, Algorithm C1 and Algorithm C2 with coarse mesh size $H=\sqrt{h}$ to solve two partial differential equations of second order, respectively. For the exact solver of all the nonsymmetric and/or indefinite systems of coarse 


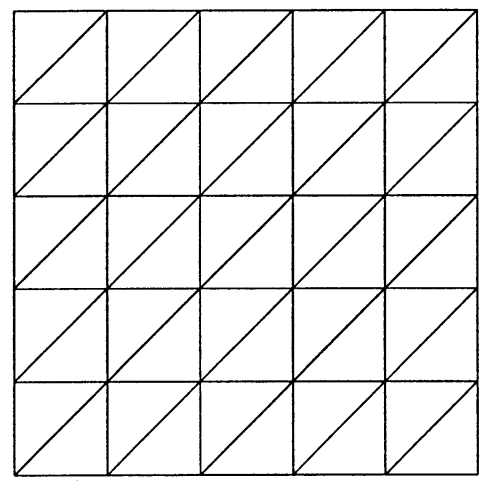

FiguRe 7. A Triangulation

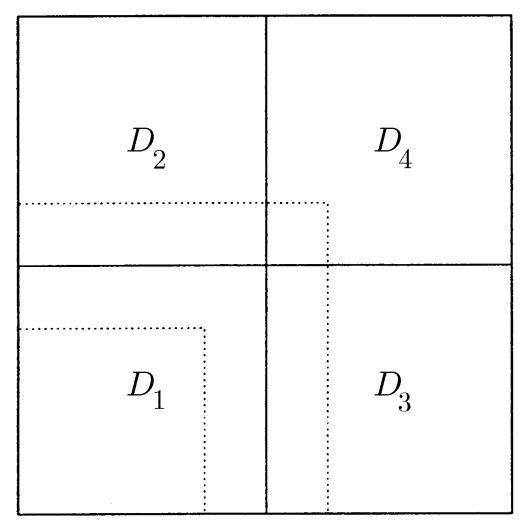

FiguRe 8. Domain Decomposition

spaces, the Gaussian elimination is used. On the other hand, a standard V-cycle multigrid algorithm is used to solve all the SPD systems on fine spaces.

We first consider the following simple Poisson equation:

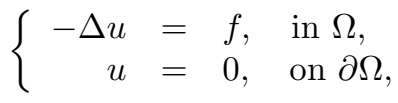

where $f=50\left(x-y^{2}\right) e^{y}$.

We shall apply Algorithm B1 and Algorithm C2 to solve this problem, using fine meshes of sizes $h=2^{-j}(j=6,8,10)$ and corresponding coarse meshes of size $H=\sqrt{h}$.

Let $u_{h}$ be the standard finite element solution, let $u^{h}$ be obtained by Algorithm B1, and let $\tilde{u}^{h}$ be obtained by Algorithm C2. Then, by Theorems 4.4 and 4.6 we obtain

$$
\left\|u_{h}-u^{h}\right\|_{1, \Omega}=\mathcal{O}\left(H^{2}\right) \approx c h, \quad\left\|u_{h}-\tilde{u}^{h}\right\|_{0, \Omega}=\mathcal{O}\left(H^{3}\right) \approx c h^{3 / 2} .
$$

The results shown in Table 1 support the above estimate. Actually, for Algorithm $\mathrm{C} 2$, this numerical example shows a better order of convergence than our theory predicted. 
TABLE 1. Algorithm B1 and Algorithm C2 for the Poisson problem

\begin{tabular}{|c|c|c|c|c|}
\hline$h$ & $\left\|u_{h}-u^{h}\right\|_{1, \Omega}$ & $\frac{\left\|u_{4 h}-u^{4 h}\right\|_{1, \Omega}}{\left\|u_{h}-u^{h}\right\|_{1, \Omega}}$ & $\left\|u_{h}-\tilde{u}^{h}\right\|_{0, \Omega}$ & $\frac{\left\|u_{4 h}-\tilde{u}^{4 h}\right\|_{0, \Omega}}{\left\|u_{h}-\tilde{u}^{h}\right\|_{0, \Omega}}$ \\
\hline $2^{-6}$ & $0.7722(-1)$ & & $0.4224(-3)$ & \\
$2^{-8}$ & $0.1948(-1)$ & 3.96 & $0.2933(-4)$ & 14.4 \\
$2^{-10}$ & $0.4885(-2)$ & 3.99 & $0.1952(-5)$ & 15.0 \\
\hline
\end{tabular}

TABle 2. Algorithm C1 and Algorithm C2 for the Non-SPD problem

\begin{tabular}{|c|c|c|c|c|}
\hline$h$ & $\left\|u_{h}-u^{h}\right\|_{1, \Omega}$ & $\frac{\left\|u_{4 h}-u^{4 h}\right\|_{1, \Omega}}{\left\|u_{h}-u^{h}\right\|_{1, \Omega}}$ & $\left\|u_{h}-\tilde{u}^{h}\right\|_{0, \Omega}$ & $\frac{\left\|u_{4 h}-\tilde{u}^{4 h}\right\|_{0, \Omega}}{\left\|u_{h}-\tilde{u}^{h}\right\|_{0, \Omega}}$ \\
\hline $2^{-6}$ & $0.1063(+0)$ & & $0.1089(-2)$ & \\
$2^{-8}$ & $0.2827(-1)$ & 3.76 & $0.1307(-3)$ & 8.33 \\
$2^{-10}$ & $0.7217(-2)$ & 3.92 & $0.1606(-4)$ & 8.14 \\
\hline
\end{tabular}

We next consider a simple example of convection-diffusion problems:

$$
\left\{\begin{aligned}
-\Delta u+\mathbf{b} \cdot \nabla u & =f, & & \text { in } \Omega, \\
u & =0, & & \text { on } \partial \Omega,
\end{aligned}\right.
$$

where $\mathbf{b}=\left(2 x-e^{y}, 3 y \cos (\pi x)\right), f=70 \log ((x+1 / 10)(\sin (\pi y)+1))$.

As for the convection-diffusion equation above, we again apply Algorithm C1 and Algorithm $\mathrm{C} 2$ to solve this problem. The corresponding computational results are shown in Table 2 and again support our theory.

\section{SOME FURTHER REMARKS}

In this last section, we shall make a few technical comments and a concluding remark.

7.1. On the dependence of subdomains. We would like to point out that most of the error estimates presented in this paper depend on the distance between the boundaries of the underlying subdomains (cf. Schatz and Wahlbin [39, 40], and Wahlbin [46, 47]). To avoid notational complication, we chose not to explicitly spell out this kind of dependence.

7.2. Estimates in terms of different norms. Most of the local estimates in this paper can be generalized to other norms such as the $W^{1, \infty}$ and $L^{\infty}$ norms. As an illustration, let us discuss briefly the possible maximum norm estimates in two dimensions.

For any $z \in \Omega$, let $G_{z}$ be the Green function with respect to the singular point $z$ :

$$
a\left(v, G_{z}\right)=v(z), \quad \forall v \in H_{0}^{1}(\Omega)
$$

We assume that

A.6. Green function.

$$
\inf _{v \in S_{0}^{h}(\Omega)}\left(\left\|h^{-2}\left(G_{z}-v\right)\right\|_{0,1, \Omega_{0}}+\left\|h^{-1}\left(G_{z}-v\right)\right\|_{1,1, \Omega_{0}}\right) \lesssim\left|\log h_{m}\right|,
$$


where $h_{m}=\min _{x \in \Omega} h(x)$. Moreover, if $z \in D \subset \subset D_{1} \subset \Omega_{0}$, then

$$
\left\|G_{z}\right\|_{2, \Omega \backslash D_{1}} \lesssim 1
$$

(The above assumption is reasonable; we refer, for example, to [17, 37] for details.) A.1" Approximation. If $w \in W_{0}^{1,1}(\Omega)$, then as $h_{\Omega} \rightarrow 0$,

$$
\inf _{v \in S_{0}^{h}(\Omega)}\left(\left\|h^{-1}(w-v)\right\|_{0,1, \Omega}+\|w-v\|_{1,1, \Omega}\right)=o(1) .
$$

A.4'. Trace.

$$
\|v\|_{0,1, \partial \tau} \lesssim\left\|h^{-1} w\right\|_{0,1, \tau}+\|\nabla w\|_{0,1, \tau}, \quad \forall w \in H^{1}(\tau), \tau \in T^{h}\left(\Omega_{0}\right) .
$$

The following theorem can be proved.

Theorem 7.1. If Assumptions A.1", A. $4^{\prime}$ and A.6 hold, then

$$
\left\|u-u_{h}\right\|_{0, \infty, D} \lesssim\left|\log h_{m}\right|\left\|h^{2} \eta\left(u_{h}\right)\right\|_{0, \infty, \Omega_{0}}+\left\|u-u_{h}\right\|_{-1, \Omega} .
$$

7.3. Improved estimates in some special cases. In our local error estimates, the global errors are all measured in $L^{2}$ norms. As in the existing literature on local a priori error estimates (cf. Schatz and Wahlbin [39, 40], and Wahlbin [46, 47]), it is possible to replace the global $L^{2}$ norm by some negative Sobolev norms. For example, the following estimate may be obtained:

$$
\left\|u-u_{h}\right\|_{1, D} \lesssim \inf _{v \in S_{0}^{h}(\Omega)}\|u-v\|_{1, \Omega_{0}}+\left\|u-u_{h}\right\|_{1-r, \Omega}
$$

for a finite element space of degree $r$. As a result, the following estimate similar to (1.3) may be obtained:

$$
\left\|u-u_{h}\right\|_{1, D}=\mathcal{O}\left(h^{r}+H^{2 r}\right) .
$$

For simplicity and generality, we did not get into details in this paper when the above improved estimates may be obtained. We will report this kind of results in our future work.

7.4. Conclusion. In this paper, we have used a simple second oder elliptic model problem and a class of finite element discretization methods to demonstrate how to use a coarse grid to capture the global component of the approximate solution and then parallelize the major computation in a much finer grid. We believe this is a general and powerful parallel-computing technique that can be used for a variety of partial differential equations with different types of discretization methods.

\section{ACKNOWLEDGMENTS}

The authors wish to thank H. Kim and L. Zikatanov for their assistance with numerical experiments and helpful discussions. This paper was completed while the first author was visiting ETH Zurich in Switzerland during the summer of 1998, and the author wishes to thank Professors C. Schwab and R. Jeltsch for their hospitality.

\section{REFERENCES}

[1] Adams R.A.(1975): Sobolev Spaces, Academic Press, New York. MR 56:9247

[2] Ainsworth, M. and Oden, J.T.(1993): A unified approach to a posteriori error estimation using element residual methods, Numer. Math., 65, 23-50. MR 95a:65185

[3] Axelsson, O. and Layton, W.(1996): A two-level discretization of nonlinear boundary value problems, SIAM J. Numer. Anal., 33, 2359-2374. MR 98c:65181 
[4] Babuska, I., Duran, R. and Rodriguez, R.(1992): Analysis of the efficiency of an posteriori error estimator for linear triangular finite elements, SIAM J. Numer. Anal., 29, 947-946. MR 93d:65096

[5] Babuska, I. and Rheinboldt, C.(1978): Error estimates for adaptive finite element computations, SIAM J. Numer. Anal., 15, 736-754. MR 58:3400

[6] Babuska, I., Zienkiewicz, O.C., Gago, J. and Oliveira, E.R. de A. (eds.)(1986): Accuracy Estimates and Adaptive Refinements in Finite Element Computations, Wiley, New York. MR 87j:65004

[7] Babuska, I., Strouboulis, T. and Gangaraj, S.K.(1997): A posteriori estimation of the error in the recovered derivatives of the finite element solution, Comput. Methods Appl. Mech. Engrg., 150, 369-396. CMP 98:06

[8] Babuska, I., Strouboulis, T., Gangaraj, S.K. and Upadhyay, C.S.(1997): Pollution error in the $h$-version of the finite element method and the local quality of the recovered derivatives, Comput. Methods Appl. Mech. Engrg., 140, 1-37. MR 97i:73092

[9] Babuska, I., Strouboulis, T. and Upadhyay, C.S.(1994): A model study of the quality of a posteriori error estimators for linear elliptic problems. Error estimation in the interior of patchwise uniform grids of triangles, Comput. Methods Appl.Mech. Engrg., 114, 307-378. MR 95d:65093

[10] Bank, R.E.(1996): Hierarchical bases and the finite element method, Acta Numerica, 5, 1-43. CMP 98:14

[11] Bank, R.E.(1998): A simple analysis of some a posteriori error estimates, Appl. Numer. Math., 26, 153-164. CMP 98:08

[12] Bank, R.E. and Holst, M.(1998): A new paradigm for parallel adaptive meshing algorithms (manuscript).

[13] Bank, R.E. and Smith, R.K.(1993): A posteriori error estimates based on hierarchical bases, SIAM J. Numer. Anal., 30, 921-935. [MR 95f:65212

[14] Bank, R.E. and Smith, R.K.(1997): Mesh smoothing using a posteriori error estimates, SIAM J. Numer. Anal., 34, 979-997. MR 98M:65162

[15] Bank, R.E. and Weiser, A.(1985): Some a posteriori error estimates for elliptic partial differential equations, Math. Comp., 44, 283-301. MR 86g:65207

[16] Bedivan, D.M.(1995): A two-grid method for solving elliptic problems with inhomogeneous boundary conditions, Comput. Math. Appl., 29, 59-66. MR 95k:65103

[17] Blum, H., Lin, Q. and Rannacher, R.(1986): Asymptotic error expansion and Richardson extrapolation for linear finite elements, Numer. Math., 49, 11-38. MR 87m:65172

[18] Bornemann, F.A., Erdmann, B. and Kornhuber, R.(1996): A posteriori error estimates for elliptic problems in two and three space dimensions, SIAM J. Numer. Anal., 33, 1188-1204. MR 98a:65161

[19] Bramble, J.H.(1993): Multigrid Methods, Pitman Research Notes in Mathematics, 294, London Co-published in the USA with Wiley, New York. MR 95b:65002

[20] Bramble, J.H., Ewing, R.E., Parashkevov, R.R. and Pasciak, J.E.(1992): Domain decomposition methods for problems with partial refinement, SIAM J. Sci. Stat. Comp., 13, 397-410. MR 92i:65179

[21] Bramble, J.H., Ewing, R.E., Pasciak, J.E. and Schatz, A.H.(1988): A preconditioning technique for the efficient solution of problems with local grid refinement, Comp. Meth. Appl. Mech. Eng., 67, 149-159.

[22] Chan, T. and Mathew, T.(1994): Domain decomposition algorithms, Acta Numerica, 3, 61-143. MR 95f:65214

[23] Ciarlet, P.G. and Lions J.L.(1991): Handbook of Numerical Analysis, Vol.II, Finite Element Methods (Part I), North-Holland. MR 92f:65001

[24] Dawson, C.N. and Wheeler, M.F.(1994): Two-grid methods for mixed finite element approximations of nonlinear parabolic equations, Contemp. Math., 180, 191-203. MR 95j:65117

[25] Dawson, C.N., Wheeler, M.F. and Woodward, C.S.(1998): A two-grid finite difference scheme for nonlinear parabolic equations, SIAM J. Numer. Anal., 35, 435-452. MRR 99b:65097

[26] Eriksson, K., Estep, D., Hansbo, P. and Johnson, C.(1995): Introduction to adaptive methods for differential equations, Acta Numerica, 105-158. MR 96k:65057

[27] Eriksson, K., Estep, D., Hansbo, P. and Johnson, C.(1996): Computational Differential Equations, Cambridge University Press. MR 97m:65006 
[28] Eriksson, K. and Johnson, C.(1991): Adaptive finite element methods for parabolic problems I: a linear model problem, SIAM J. Numer. Anal., 28, 43-77. MR 91m:65274

[29] Eriksson, K. and Johnson, C.(1995): Adaptive finite element methods for parabolic problems IV: Nonlinear problems, SIAM J. Numer. Anal., 32, 1729-1749. MR 96i:65081

[30] Grisvard, P.(1985): Elliptic Problems in Nonsmooth Domains, Pitman, Boston, MA. MR 86m:35044

[31] Hackbusch, W.(1985): Multigrid Methods and Applications, Springer, New York. MR 87e:65082

[32] Johnson, C.(1990): Adaptive finite element methods for diffusion and convection problems, Comp. Methods Appl. Mech. Engrg., 82, 301-322. MR 91k:65134

[33] Layton, W. and Lenferink, W.(1995): Two-level Picard and modified Picard methods for the Navier-Stokes equations, Appl. Math. Comp., 69, 263-274. MR 95m:65191

[34] Marion, M. and Xu, J.(1995): Error estimates on a new nonlinear Galerkin method based on two-grid finite elements, SIAM J. Numer. Anal., 32, 1170-1184. MR 96f:65136

[35] Nitsche, J. and Schatz, A.H.(1974): Interior estimates for Ritz-Galerkin methods, Math. Comp., 28, 937-955. MR 51:9525

[36] Nochetto, R. H.(1995): Pointwise a posteriori error estimates for elliptic problems on highly graded meshes, Math. Comp., 64, 1-22. MR 95c:65172

[37] Rannacher, R. and Scott, R.(1982): Some optimal error estimates for piecewise linear finite element approximations, Math. Comp., 38, 437-445. MR 83e:65180

[38] Schatz, A.H.(1998): Pointwise error estimates and asymptotic error expansion inequalities for the finite element method on irregular grids: Part I. Global estimates, Math. Comp., 67, 877-899. MR 98j:65082

[39] Schatz, A.H. and Wahlbin, L.B.(1977): Interior maximum-norm estimates for finite element methods, Math. Comp., 31, 414-442. MR 55:4748

[40] Schatz, A.H. and Wahlbin, L.B.(1995): Interior maximum-norm estimates for finite element methods, Part II, Math. Comp., 64, 907-928. MR 95j:65143

[41] Schatz, A.H. and Wang, J.(1996): Some new error estimates for Ritz-Galerkin methods with minimal regularity assumptions, Math. Comp., 65, 19-27. MR 96d:65190

[42] Utnes, T.(1997): Two-grid finite element formulations of the incompressible Navier-Stokes equations, Comm. Numer. Methods Engrg., 34, 675-684. MR 98d:76110

[43] Verfürth, R.(1994): A posteriori error estimates for nonlinear problems. Finite element discretizations of elliptic equations, Math. Comp., 62, 445-475. MR 94j:65136

[44] Verfürth, R.(1995): A posteriori error estimates for nonlinear problems. Finite element discretizations of parabolic equations, Bericht Nr. 180, Fakultät für Mathematik, RuhrUniversität Bochum.

[45] Verfürth, R.(1996): A Review of A-Posteriori Error Estimation and Adaptive Mesh Refinement, Wiley-Teubner.

[46] Wahlbin, L.B.(1991): Local behavior in finite element methods, in 23, pp. 355-522. MR 92f: 65001

[47] Wahlbin, L.B.(1995): Superconvergence in Galerkin Finite Element Methods, Vol. 1605, Lecture Notes in Math., Springer. MR 98j:65083

[48] Xu, J.(1992): A new class of iterative methods for nonselfadjoint or indefinite problems, SIAM J. Numer. Anal., 29, 303-319. MR 92k:65063

[49] Xu, J.(1992): Iterative methods by space decomposition and subspace correction, SIAM Review, 34, 4, 581-613. MR 93k:65029

[50] Xu, J.(1994): A novel two-grid method for semilinear equations, SIAM J. Sci. Comput., 15, 231-237. MR 94m:65178

[51] Xu, J.(1996): Two-grid discretization techniques for linear and nonlinear PDEs, SIAM J. Numer. Anal., 33, 1759-1777. MR 97i:65169

[52] Xu, J. and Zou, J.(1998): Some non-overlapping domain decomposition methods, SIAM Review 40, 4, 857-914.

[53] Yserentant, H.(1993): Old and new proofs for multigrid algorithms, Acta Numerica, 2, 285326. MR 94i:65128

[54] Zhou, A., Liem, C.L., Shih, T.M. and Lü, T.(1998): Error analysis on bi-parameter finite elements, Comput. Methods Appl. Mech. Engrg., 158, 329-339. CMP 98:14 
Center for Computational Mathematics and Applications, Department of Mathematics, Pennsylvania State University, University Park, Pennsylvania 16802

E-mail address: xu@math.psu.edu

Institute of Systems Science, Academia Sinica, Beijing 100080, China

E-mail address: azhou@bamboo.iss.ac.cn 